\title{
Anisotropic Polyoxometalate Cages Assembled via Layers of Heteroanion Templates
}

Qi Zheng, ${ }^{[\mathrm{a}]}$ Manuel Kupper, ${ }^{[\mathrm{a}]}$ Weimin Xuan, ${ }^{[\mathrm{a}]}$ Hirofumi Oki, ${ }^{[\mathrm{b}]}$ Ryo Tsunashima, ${ }^{[\mathrm{b}]}$ De-Liang Long*[a] and Leroy Cronin*[a]

[a] School of Chemistry, The University of Glasgow, Glasgow G12 8QQ, UK

${ }^{[b]}$ Graduate School of Sciences and Technology for Innovation, Yamaguchi University, Yoshida 1677-1, Yamaguchi, 7538512, Japan

\section{Table of Contents}

1. Materials and instrumentation $\quad$ S2

2. Synthetic procedures $\quad$ S3

3. Single-crystal X-ray structure determinations $\quad$ S6

4. UV-Vis-NIR spectroscopy $\quad$ S11

5. ESI-Mass spectrometry $\quad$ S12

6. NMR spectroscopy $\quad \mathrm{S} 14$

7. Dielectric studies $\quad$ S15

8. TGA analysis $\quad \mathrm{S} 17$

9. Redox titrations $\quad$ S22

10. References $\quad$ S22 


\section{Materials and instrumentation}

Materials.

Reagent-grade chemicals were obtained from Aldrich Chemical Company Ltd. and Alfa Aesar, and used without further purification.

\section{Instrumentation.}

Single Crystal X-ray Diffraction: Suitable single crystals were selected and mounted by using the MiTeGen MicroMountsTM kit with Fomblin oil. X-ray diffraction intensity data were measured at 150(2) K on Bruker Apex II Quazar diffractometers using Mo $\mathrm{Ka}[\lambda=0.71073 \AA]$. Structure solution and refinement were carried out with SHELXS ${ }^{[1]}$ and SHELXL-2014 ${ }^{[2]}$ via WinGX ${ }^{[3]}$. Corrections for incident and diffracted beam absorption effects were applied using empirical methods ${ }^{[4]}$. The $X$-ray crystallographic data for structures reported in this article have been deposited at the Cambridge Crystallographic Data Centre, under deposition number CCDC-1885487-1885496. These data can be obtained free of charge via www.ccdc.cam.ac.uk/data_request/cif, or by emailing data_request@ccdc.cam.ac.uk, or by contacting The Cambridge Crystallographic Data Centre, 12 Union Road, Cambridge CB2 1EZ, UK; fax: +44 1223336033.

UV-Vis-NIR spectroscopy: UV-Vis-NIR spectroscopy experiments were carried out using a SPECORD S600 Analytic Jena spectrophotometer. The measurements were performed in a quartz glass cuvette with optical path length $2.0 \mathrm{~mm}$.

Microanalysis: Carbon, nitrogen and hydrogen content were determined by the microanalysis services within the Department of Chemistry, University of Glasgow using an EA 1110 CHNS, CE-440 Elemental Analyzer.

Inductively Coupled Plasma Optical Emission Spectroscopy (ICP-OES): Samples were prepared in $3 \mathrm{~mL} \mathrm{HNO}_{3} / 2 \mathrm{~mL} \mathrm{H}_{2} \mathrm{O}_{2}$ and diluted with water to make $50 \mathrm{~mL}$ total volume. The relative error in the measurements is $\pm 3 \%$.

Fourier-transform infrared (FT-IR) spectroscopy: The sample was prepared as a $\mathrm{KBr}$ pellet and the FT IR spectrum was collected in transmission mode in the range of $400-4000 \mathrm{~cm}^{-1}$ using a JASCO FT-IR 4100 spectrometer. Wavenumbers are given in $\mathrm{cm}^{-1}$.

Thermogravimetric Analysis (TGA): Thermogravimetric analysis was performed on a TA Instruments Q 500 Thermogravimetric Analyzer under nitrogen flow at a typical heating rate of $10^{\circ} \mathrm{C} \mathrm{min}^{-1}$.

Redox titration: Redox titration of the compounds was performed by using cerium ammonium nitrate $(0.0025 \mathrm{M})$ in $0.5 \mathrm{M} \mathrm{H}_{2} \mathrm{SO}_{4}$ as the oxidant.

Electrospray-ionization mass spectrometry (ESI-MS): All MS data was collected using a Q-trap, time-of-flight MS (Maxis Impact) instrument supplied by Bruker Daltonics Ltd. A Bruker Ltd cryospray source was used to collect data under the conditions specified below. The detector was a time-of-flight, micro-channel plate detector and all data was processed using the BrukerDaltonics Data Analysis 4.1 software, whilst simulated isotope patterns were investigated using Bruker Isotope Pattern software and Molecular Weight Calculator 6.45. All peak assignments were made via comparison of the experimentally determined isotopic patterns for each peak with simulated isotopic patterns. The sample solutions of 1-3 were prepared by dissolving the compounds in a water/ acetonitrile (5\%:95\%) mixture $\left(1 \mathrm{mg} \mathrm{mL}^{-1}\right)$ and sample solution of 11 was prepared by dissolving the compounds in acetonitrile $1 \mathrm{mg} \mathrm{mL}^{-1}$.

Nuclear Magnetic Resonance Spectroscopy: ${ }^{125} \mathrm{Te}$ NMR spectroscopy were recorded on Bruker AVIII 600MHz spectrometer.

Dielectric measurement: Temperature-dependent dielectric permittivity was measured using an Agilent E4980A Precision LCR meter between 100 and $310 \mathrm{~K}$ controlled at $0.5 \mathrm{~K} / \mathrm{min}$. The pellets were made from a powdered sample after drying. The typical applied AC voltage was $1 \mathrm{~V}$, and the frequency range was $100 \mathrm{~Hz}-1 \mathrm{MHz}$. 


\section{Synthetic procedure:}

\section{Synthesis of $\left[\mathrm{Mov}_{2} \mathrm{O}_{4}\left(\mathrm{H}_{2} \mathrm{O}\right)_{2}\right]^{2+}$ in $4 \mathrm{M} \mathrm{HCl}\left(\left\{\mathrm{Mo}_{2}\right\}\right)$}

The synthesis is based on reported procedure. ${ }^{[5]} 840 \mu \mathrm{L}$ of hydrazine monohydrate $(64-65 \%)$ was added to a suspension of 9.2 $\mathrm{g}$ molybdenum trioxide in $320 \mathrm{~mL} 4 \mathrm{M} \mathrm{HCl}$. The solution was heated at $60^{\circ} \mathrm{C}$ for 3 hours.

\section{Synthesis of $\left(\mathrm{C}_{2} \mathrm{H}_{8} \mathrm{~N}\right)_{7} \mathrm{H}_{3}\left[\mathrm{HPW}_{16} \mathrm{Mo}_{2} \mathrm{O}_{60}\right] \cdot 10 \mathrm{H}_{2} \mathrm{O}$ (1)}

Sodium tungstate dihydrate $(2.5 \mathrm{~g}, 7.6 \mathrm{mmol})$, phosphorous acid $(0.04 \mathrm{~g}, 0.48 \mathrm{mmol})$ and dimethylamine hydrochloride (DMA. $\mathrm{HCl})$ $(1.2 \mathrm{~g}, 14.7 \mathrm{mmol})$ were dissolved in $20 \mathrm{~mL}$ water. Around $2.8 \mathrm{~mL}$ of $\left\{\mathrm{Mo}_{2}\right\}$ was added dropwise under stirring, and the solution $\mathrm{pH}$ consequently reached 2.2. The solution was heated with stirring at around $50^{\circ} \mathrm{C}$ for $30 \mathrm{mins}$. Cool down to room temperature, dark blue powder formed and was removed by filtration. Dark blue block crystals were isolated from the filtrate after a few days. These crystals were collected by soaking the solid using filtered mother liquid to remove further blue powder (Yield: $8.3 \%$ based on W). Characteristic IR bands ( $\left.\mathrm{cm}^{-1}\right)$ : 3419(b), 3151(b), 2980(s), 2785(w), 2451(w), 1614(m), 1462(s), 1379(m), 1249(w), 1153(w) 1066(m), 951(s), 721(s), 501(w). Elemental Analysis for $\mathrm{C}_{14} \mathrm{H}_{80} \mathrm{~N}_{7} \mathrm{P}_{1} \mathrm{~W}_{16} \mathrm{Mo}_{2} \mathrm{O}_{70}, \mathrm{MW}_{\mathrm{W}}=4631.23 \mathrm{~g} \mathrm{~mol}^{-1}$, calc (\%): W 63.51, Mo 4.14, P 0.67, H 1.74, C 3.63, N 2.11; found (\%): W 64.09, Mo 3.87, P 0.76, H 1.63, C 3.14, N 2.32. Calculated TGA water loss from 25 to $200{ }^{\circ} \mathrm{C}(\%)$ calculated: 3.9 , found 3.1 .

\section{Synthesis of $\left(\mathrm{C}_{2} \mathrm{H}_{8} \mathrm{~N}\right)_{7} \mathrm{H}_{3}\left[\mathrm{SeW}_{16} \mathrm{Mo}_{2} \mathrm{O}_{60}\right] \cdot 10 \mathrm{H}_{2} \mathrm{O}(2)$}

Sodium tungstate dihydrate $(2.5 \mathrm{~g}, 7.6 \mathrm{mmol})$, sodium selenite $(0.08 \mathrm{~g}, 0.46 \mathrm{mmol})$ and dimethylamine hydrochloride (DMA.HCl) $(1.2 \mathrm{~g}, 14.7 \mathrm{mmol})$ were dissolved in $20 \mathrm{~mL}$ water. 2.8-3.4 $\mathrm{mL}$ of $\left\{\mathrm{Mo}_{2}\right\}$ was added dropwise under stirring, and the solution $\mathrm{pH}$ consequently reached 1.3-2.6. The solution was heated with stirring at around $50{ }^{\circ} \mathrm{C}$ for $30 \mathrm{mins}$. Cool down to room temperature, dark blue powder formed and was removed by filtration. Dark blue block crystals were isolated from the filtrate after one week. These crystals were collected by soaking the solid using filtered mother liquid to remove further blue powder (Yield: $22.4 \%$ based on W). Characteristic IR bands $\left(\mathrm{cm}^{-1}\right)$ : 3452(b), 3140(b), 2980(s), 2779(m), 2453(w), 1614(m), 1462(s), 1388(m), 1253(w), 1161(s) 1068(m), 1018(m), 950(s), 704(s). Elemental Analysis for $\mathrm{C}_{14} \mathrm{H}_{79} \mathrm{~N}_{7} \mathrm{Se}_{1} \mathrm{~W}_{16} \mathrm{Mo}_{2} \mathrm{O}_{70}, \mathrm{MW}_{\mathrm{W}}=4678.21 \mathrm{~g} \mathrm{~mol}^{-1}$, calc (\%): W 62.87, Mo 4.10, Se 1.68, H 1.70, C 3.59, N 2.09; found (\%): W 57.36, Mo 3.19, Se 1.77, H 1.56, C 3.53, N 2.03. Calculated TGA water loss from 25 to $200{ }^{\circ} \mathrm{C}(\%)$ calculated: 3.8 , found 3.3 .

\section{Synthesis of $\left(\mathrm{C}_{2} \mathrm{H}_{8} \mathrm{~N}\right)_{7} \mathrm{H}_{3}\left[\mathrm{TeW}_{16} \mathrm{Mo}_{2} \mathrm{O}_{60}\right] \cdot 10 \mathrm{H}_{2} \mathrm{O}(3)$}

Sodium tungstate dihydrate $(2.5 \mathrm{~g}, 7.6 \mathrm{mmol})$, sodium tellurite $(0.1 \mathrm{~g}, 0.48 \mathrm{mmol})$ and dimethylamine hydrochloride (DMA.HCl) $(1.2 \mathrm{~g}, 14.7 \mathrm{mmol})$ were dissolved in $20 \mathrm{~mL}$ water. Around $3 \mathrm{~mL}$ of $\left\{\mathrm{Mo}_{2}\right\}$ was added dropwise under stirring, and the solution $\mathrm{pH}$ consequently reached 1.9 . The solution was heated with stirring at around $50{ }^{\circ} \mathrm{C}$ for 30 mins. Cool down to room temperature, big dark blue block crystals were isolated from the filtrate after one week. (Yield: $62.5 \%$ based on W). Characteristic IR bands (cm 1): 3448(b), 3159(b), 3035(b), 2786(m), 2447(w), 1614(m), 1463(s), 1244(w), 1071(m), 957(s), 877(s), 713(s), 487(w). Elemental Analysis for $\mathrm{C}_{14} \mathrm{H}_{79} \mathrm{~N}_{7} \mathrm{Te}_{1} \mathrm{~W}_{16} \mathrm{Mo}_{2} \mathrm{O}_{70}, \mathrm{M}_{\mathrm{W}}=4726.85 \mathrm{~g} \mathrm{~mol}^{-1}$, calc (\%): W 62.23, Mo 4.06, Te 2.70, H 1.68, C 3.56, N 2.07; found (\%): W 61.87, Mo 4.28, Te 2.76, H 1.77, C 3.93, N 2.26. Calculated TGA water loss from 25 to $200{ }^{\circ} \mathrm{C}(\%)$ calculated: 3.8 , found 1.8.

\section{Synthesis of $\left(\mathrm{C}_{2} \mathrm{H}_{8} \mathrm{~N}\right){ }_{11} \mathrm{NaH}\left[\mathrm{H}_{2} \mathrm{P}_{2} \mathrm{~W}_{21} \mathrm{Mo}_{3} \mathrm{O}_{81}\right] \cdot 15 \mathrm{H}_{2} \mathrm{O}$ (4)}

Sodium tungstate dihydrate $(1.6 \mathrm{~g}, 4.8 \mathrm{mmol})$, phosphorous acid $(0.04 \mathrm{~g}, 0.48 \mathrm{mmol})$ and dimethylamine hydrochloride (DMA. HCl) $(1.2 \mathrm{~g}, 14.7 \mathrm{mmol})$ were dissolved in $40 \mathrm{~mL}$ water. Around $1.6 \mathrm{~mL}$ of $\left\{\mathrm{Mo}_{2}\right\}$ was added dropwise under stirring, and the solution $\mathrm{pH}$ consequently reached 3.3. The solution was heated with stirring at around $50^{\circ} \mathrm{C}$ for $30 \mathrm{mins}$. Cool down to room temperature, dark blue powder formed and was removed by filtration. Dark blue precipitates appear after a few days and were removed by filtration. Small dark blue star or rod-like crystals were isolated after three weeks. These crystals were collected by soaking the solid using filtered mother liquid to remove further blue powder (Yield: $6.3 \%$ based on W). Characteristic IR bands $\left(\mathrm{cm}^{-1}\right): 3448(\mathrm{~b})$, 3140(b), 2980(s), 2787(w), 2455(w), 1614(m), 1462(s), 1381(m), 1251(m), 1149(s), 1066(m), 952(s), 731(s). Elemental Analysis for $\mathrm{C}_{22} \mathrm{H}_{121} \mathrm{~N}_{11} \mathrm{Na}_{1} \mathrm{P}_{2} \mathrm{~W}_{21} \mathrm{Mo}_{3} \mathrm{O}_{96}, \mathrm{M}_{\mathrm{W}}=6309.8 \mathrm{~g} \mathrm{~mol}^{-1}$, calc (\%): W 61.19, Mo 4.56, P 0.98, Na 0.36, H 1.93, C 4.19, N 2.44; found (\%): W 59.66, Mo 4.24, P 0.76, Na 0.79, H 1.80, C 4.06, N 2.33. Calculated TGA water loss from 25 to $200{ }^{\circ} \mathrm{C}(\%)$ calculated: 4.3 , found 2.8

\section{Synthesis of $\left(\mathrm{C}_{2} \mathrm{H}_{8} \mathrm{~N}\right)_{11} \mathrm{NaH}_{3}\left[\mathrm{Se}_{2} \mathrm{~W}_{21} \mathrm{Mo}_{3} \mathrm{O}_{82}\right] \cdot 14 \mathrm{H}_{2} \mathrm{O}(5)$}

Sodium tungstate dihydrate $(1.36 \mathrm{~g}, 4.1 \mathrm{mmol})$, sodium selenite $(0.08 \mathrm{~g}, 0.46 \mathrm{mmol})$ and dimethylamine hydrochloride (DMA.HCl) $(1.2 \mathrm{~g}, 14.7 \mathrm{mmol})$ were dissolved in $20 \mathrm{~mL}$ water. Around $1.5 \mathrm{~mL}$ of $\left\{\mathrm{Mo}_{2}\right\}$ was added dropwise under stirring, and consequently the solution $\mathrm{pH}$ reached 2.2. The solution was heated with stirring at around $50{ }^{\circ} \mathrm{C}$ for $30 \mathrm{mins}$. Cool down to room temperature, dark blue powder formed and was removed by filtration. Blue precipitates appear constantly, and were removed by filtration. Small dark blue needle-shaped crystals were isolated from the filtrate after three months. These crystals were collected by soaking the solid using filtered mother liquid to remove further blue powder (Yield: $8.0 \%$ based on W). Characteristic IR bands $\left(\mathrm{cm}^{-1}\right): 3431(\mathrm{~b})$, 3159(b), 2980(m), 2879(w), 2786(w), 2436(w), 1614(m), 1462(m), 1379(m), 1253(m), 1153(m), 1070(m), 952(s), 746(s), 488(w). Elemental Analysis for $\mathrm{C}_{22} \mathrm{H}_{119} \mathrm{~N}_{11} \mathrm{Na}_{1} \mathrm{Se}_{2} \mathrm{~W}_{21} \mathrm{Mo}_{3} \mathrm{O}_{96}, \mathrm{M}_{\mathrm{W}}=6403.60 \mathrm{~g} \mathrm{~mol}^{-1}$, calc (\%): W 60.29, Mo 4.49, Se 2.47, Na 0.36, H 1.87, C 4.12, N 2.41; found (\%): W 61.87, Mo 4.48, Se 1.90, Na 0.34, H 1.71, C 4.60, N 2.40. Calculated TGA water loss from 25 to $200{ }^{\circ} \mathrm{C}(\%)$ calculated: 3.9 , found 3.2 .

\section{Synthesis of $\left(\mathrm{C}_{2} \mathrm{H}_{8} \mathrm{~N}\right){ }_{10} \mathrm{NaH}_{3}\left[\mathrm{Te}_{2} \mathrm{~W}_{22} \mathrm{Mo}_{2} \mathrm{O}_{82}\right] \cdot 14 \mathrm{H}_{2} \mathrm{O}(6)$}


Sodium tungstate dihydrate $(7.5 \mathrm{~g}, 22.75 \mathrm{mmol})$, sodium tellurite $(0.3 \mathrm{~g}, 1.35 \mathrm{mmol})$ and dimethylamine hydrochloride (DMA.HCl) $(3.6 \mathrm{~g}, 44.1 \mathrm{mmol})$ were dissolved in $80 \mathrm{~mL}$ water. Around $9.2 \mathrm{~mL}$ of $\left\{\mathrm{Mo}_{2}\right\}$ was added dropwise under stirring, and consequently, the solution $\mathrm{pH}$ reached 2.5. The solution was heated with stirring at around $50{ }^{\circ} \mathrm{C}$ for $30 \mathrm{mins}$. Cool down to room temperature, dark blue powder formed and was removed by filtration. Compound 3 formed and were isolated after a few days. Afterwards, blue precipitates appear constantly, and were removed by filtration. Finally, small dark blue rod-like crystals were isolated from the filtrate after four months. These crystals were collected by soaking the solid using filtered mother liquid to remove further blue powder (Yield: 7.5\% based on W). Characteristic IR bands (cm $\left.{ }^{-1}\right):$ 3444(b), 3143(b), 2981(m), 2783(w), 2443(w), 1608(m), 1463(s), 1382(w), 1247(w), 155(w), 1082(m), 1014(m), 954(s), 858(w), 725(s), 472(w). Elemental Analysis for $\mathrm{C}_{20} \mathrm{H}_{111} \mathrm{~N}_{10} \mathrm{Na}_{1} \mathrm{Te}_{2} \mathrm{~W}_{22} \mathrm{Mo}_{2} \mathrm{O}_{96}, \mathrm{MW}_{\mathrm{W}}=6542.65 \mathrm{~g} \mathrm{~mol}^{-1}$, calc (\%): W 61.81, Mo 2.93, Te 3.90, Na 0.35, H 1.71, C 3.67, N 2.14; found (\%): W 62.73, Mo 3.49, Te 4.82, Na 0.60, H 1.62, C 4.14, N 2.50. Calculated TGA water loss from 25 to $200{ }^{\circ} \mathrm{C}$ (\%) calculated: 3.8, found 2.5 .

\section{Synthesis of $\left(\mathrm{C}_{2} \mathrm{H}_{8} \mathrm{~N}\right)_{10} \mathrm{NaH}\left[\mathrm{HPSeW}{ }_{22} \mathrm{Mo}_{2} \mathrm{O}_{81}\right] \cdot 15 \mathrm{H}_{2} \mathrm{O}(7)$}

Sodium tungstate dihydrate $(2.5 \mathrm{~g}, 7.6 \mathrm{mmol})$, phosphorous acid $(0.02 \mathrm{~g}, 0.24 \mathrm{mmol})$, sodium selenite $(0.04 \mathrm{~g}, 0.23 \mathrm{mmol})$ and dimethylamine hydrochloride (DMA. HCl) $\left(1.2 \mathrm{~g}, 14.7 \mathrm{mmol}\right.$ ) were dissolved in $20 \mathrm{~mL}$ water. Around $2.6 \mathrm{ml}$ of $\left\{\mathrm{Mo}_{2}\right\}$ was added dropwise under stirring, and the solution $\mathrm{pH}$ consequently reached 2.0. The solution was heated with stirring at around $50^{\circ} \mathrm{C}$ for 30 mins. Cool down to room temperature, dark blue powder formed and was removed by filtration. Peanut-shaped clusters formed after a few weeks and were removed from the solution by filtration. Blue precipitates appear constantly afterward and were also removed by filtration. After two months, blue block crystals of 7 were formed and isolated. These crystals were collected by soaking the solid using filtered mother liquid to remove further blue powder (Yield:13.0\% based on P or Se). Characteristic IR bands (cm$\left.{ }^{1}\right)$ : 3430(b), 3032(b), 2758(w), 1694(m), 1464(s), 1246(w), 1064(m), 1016(m), 957(s), 892(w), 722(s), 550(w). Elemental Analysis for $\mathrm{C}_{20} \mathrm{H}_{112} \mathrm{~N}_{10} \mathrm{Na}_{1} \mathrm{Se}_{1} \mathrm{P}_{1} \mathrm{~W}_{22} \mathrm{Mo}_{2} \mathrm{O}_{96}, \mathrm{M}_{\mathrm{W}}=6398.59 \mathrm{~g} \mathrm{~mol}^{-1}$, calc (\%): W 63.21, Mo 3.00, P 0.48, Se 1.23, Na 0.36, H 1.76, C 3.75, N 2.19; found (\%): W 59.12, Mo 3.63, P 0.87, Se 1.54, Na 0.25, H 1.75, C 3.87 N 2.20. Calculated TGA water loss from 25 to $200^{\circ} \mathrm{C}(\%)$ calculated: 4.2 , found 4.1 .

\section{Synthesis of $\left(\mathrm{C}_{2} \mathrm{H}_{8} \mathrm{~N}\right){ }_{11} \mathrm{NaH}\left[\mathrm{HPTeW}{ }_{21} \mathrm{Mo}_{3} \mathrm{O}_{81}\right] \cdot 15 \mathrm{H}_{2} \mathrm{O}(8)$}

Sodium tungstate dihydrate $(2.5 \mathrm{~g}, 7.6 \mathrm{mmol})$, phosphorous acid $(0.02 \mathrm{~g}, 0.24 \mathrm{mmol})$, sodium tellurite $(0.05 \mathrm{~g}, 0.24 \mathrm{mmol})$ and dimethylamine hydrochloride (DMA $\mathrm{HCl})(1.2 \mathrm{~g}, 14.7 \mathrm{mmol})$ were dissolved in $20 \mathrm{~mL}$ water. Around $2.4 \mathrm{~mL}$ of $\left\{\mathrm{Mo}_{2}\right\}$ was added dropwise under stirring, and the solution $\mathrm{pH}$ consequently reached 3.3. The solution was heated with stirring at around $50{ }^{\circ} \mathrm{C}$ for 30 mins. Cool down to room temperature, dark blue powder formed and was removed by filtration. Peanut-shaped clusters formed after a few weeks and were removed from the solution by filtration. Blue precipitates appear constantly afterwards and were also removed by filtration. Dark blue block crystals were isolated from the filtrate after six weeks. These crystals were collected by soaking the solid using filtered mother liquid to remove further blue powder (Yield: $20.0 \%$ based on P). Characteristic IR bands $\left(\mathrm{cm}^{-1}\right)$ : 3448(b), 2958(w), 3035(b), 2872(w), 2764(m), 2420(w), 1596(m), 1463(s), 1410(w), 1379(w), 1067(m), 1016(m), 958(s), 884(s), 736(s), 563(w). Elemental Analysis for $\mathrm{C}_{20} \mathrm{H}_{102} \mathrm{~N}_{10} \mathrm{Na}_{1} \mathrm{P}_{1} \mathrm{Te}_{1} \mathrm{~W}_{21} \mathrm{Mo}_{3} \mathrm{O}_{91}, \mathrm{MW}_{\mathrm{W}}=6269.25 \mathrm{~g} \mathrm{~mol}^{-1}$, calc (\%): W 61.58, Mo 4.59, P 0.49, Te 2.04, Na 0.37, H 1.64, C 3.83, N 2.23; found (\%): W 55.1, Mo 4.19, P 0.64, Te 2.5, Na 0.29, H 1.57, C 3.70, N 2.10. Calculated TGA water loss from 25 to $200{ }^{\circ} \mathrm{C}(\%)$ calculated: 4.3 , found 2.6 .

\section{Synthesis of $\left(\mathrm{C}_{2} \mathrm{H}_{8} \mathrm{~N}\right){ }_{16}\left[\mathrm{H}_{3} \mathrm{P}_{3} \mathrm{~W}_{26} \mathrm{Mo}_{4} \mathrm{O}_{102}\right] \cdot 15 \mathrm{H}_{2} \mathrm{O}(9)$}

Sodium tungstate dihydrate $(1.6 \mathrm{~g}, 4.8 \mathrm{mmol})$, phosphorous acid $(0.04 \mathrm{~g}, 0.48 \mathrm{mmol})$ and dimethylamine hydrochloride (DMA.HCl) $(1.2 \mathrm{~g}, 14.7 \mathrm{mmol})$ were dissolved in $40 \mathrm{~mL}$ water. Around $1.6 \mathrm{ml}$ of $\left\{\mathrm{Mo}_{2}\right\}$ was added dropwise under stirring, and the solution $\mathrm{pH}$ consequently reached 3.2. The solution was heated with stirring at around $65^{\circ} \mathrm{C}$ for 30 mins. Cool down to room temperature, dark blue powder formed and was removed by filtration. Blue precipitates appear constantly afterwards and were also removed by filtration. Dark blue bar-shaped crystals of $\mathbf{9}$ mixed with $\mathbf{4}$ were found from the filtrate after two months.

\section{Synthesis of $\left(\mathrm{C}_{2} \mathrm{H}_{8} \mathrm{~N}\right){ }_{30} \mathrm{NaH}\left[\mathrm{Se}_{3} \mathrm{~W}_{26} \mathrm{Mo}_{4} \mathrm{O}_{102}\right]\left[\mathrm{Se}_{4} \mathrm{~W}_{36} \mathrm{O}_{124}\right] \cdot 40 \mathrm{H}_{2} \mathrm{O}$ (10)}

Sodium tungstate dihydrate $(1.6 \mathrm{~g}, 4.8 \mathrm{mmol})$, sodium selenite $(0.08 \mathrm{~g}, 0.46 \mathrm{mmol})$ and dimethylamine hydrochloride (DMA.HCl) $(1.2 \mathrm{~g}, 14.7 \mathrm{mmol})$ were dissolved in $40 \mathrm{~mL}$ water. Around $2.1 \mathrm{~mL}$ of $\left\{\mathrm{Mo}_{2}\right\}$ was added dropwise under stirring, and consequently the solution $\mathrm{pH}$ reached 2.4. The solution was heated with stirring at around $65^{\circ} \mathrm{C}$ for 30 mins. Cool down to room temperature, dark blue powder formed and was removed by filtration. Precipitates formed constantly and were removed by filtration. Then, pale blue precipitates together with "Trojan Horse"-type $\left[\mathrm{Se}_{2} \mathrm{~W}_{18} \mathrm{O}_{62}\left(\mathrm{H}_{2} \mathrm{O}\right)_{2}\right]^{8-[6]}$ appear. Dark blue needle-shaped crystals of 10 were isolated from the filtrate after two months. These crystals were collected by soaking the solid using filtered mother liquid to remove further blue powder (Yield: 11.4\% based on Se). Characteristic IR bands $\left(\mathrm{cm}^{-1}\right)$ : 3455(b), 3137(b), 2786(w), 1605(m), 1464(m), 1016(m), 951(s), 714(s), 646(s). Elemental Analysis for $\mathrm{C}_{60} \mathrm{H}_{331} \mathrm{~N}_{30} \mathrm{Na}_{1} \mathrm{Se}_{7} \mathrm{~W}_{62} \mathrm{Mo}_{4} \mathrm{O}_{271}, \mathrm{M}_{\mathrm{W}}=18077.8 \mathrm{~g} \mathrm{~mol}{ }^{-1}$, calc (\%): W 63.05, Mo 2.12, Se 3.06, Na 0.12, H 1.78, C 3.99, N 2.32; found (\%): W 58.89, Mo 2.13, Se 3.31, Na 0.17, H 1.62, C 4.15, N 2.30. Calculated TGA water loss from 25 to $200^{\circ} \mathrm{C}(\%)$ calculated: 4.0 , found 3.7 .

\section{Synthesis of $\left(\mathrm{C}_{16} \mathrm{H}_{36} \mathrm{~N}\right)_{5}\left[\mathrm{H}_{3} \mathrm{TeW}_{16} \mathrm{Mo}_{2} \mathrm{O}_{60}\right]$ (11)}

$2.0 \mathrm{~g}$ of compound $3(0.42 \mathrm{mmol})$ was dissolved in $25 \mathrm{~mL}$ of $0.5 \mathrm{M} \mathrm{HCl}$. Then $15 \mathrm{~mL}$ solution of $5.0 \mathrm{~g}$ of tetrabutylammonium bromide was added under vigorous stirring. Blue powder was formed and isolated by centrifugation and collected, washed with water, ethanol and then dried under vacuum. Recrystallization of the solid from acetonitrile with ether diffusion afforded yellow crystals of 11. (Yield: $37.10 \%$ based on compound 3). Characteristic IR bands $\left(\mathrm{cm}^{-1}\right): 2958(\mathrm{~m}), 2872(\mathrm{~m}), 1482(\mathrm{~s}), 1464(\mathrm{~m})$, 1378(m), 1151(w), 967(s), 951(s), 888(s), 771(s), 738(s). Elemental Analysis for $\mathrm{C}_{80} \mathrm{H}_{183} \mathrm{~N}_{5} \mathrm{Te}_{1} \mathrm{~W}_{16} \mathrm{Mo}_{2} \mathrm{O}_{60}, \mathrm{M}_{\mathrm{W}}=5436.4 \mathrm{~g} \mathrm{~mol}{ }^{-1}$, 
calc (\%): W 54.10, Mo 3.53, Te 2.35, H 3.39, C 17.67, N 1.29; found (\%): W 54.50, Mo 3.15, Te 3.50, H 3.34, C 17.83, N 1.30. Calculated TGA solvent loss from 25 to $250{ }^{\circ} \mathrm{C}(\%)$ calculated: 0.0 , found 1.0 .

\section{Control Experiments:}

The synthesis of 4 was chosen as a synthetic control experiment, following the general procedure:

Sodium tungstate dihydrate $(1.6 \mathrm{~g}, 4.8 \mathrm{mmol})$, phosphorous acid $(0.04 \mathrm{~g}, 0.48 \mathrm{mmol})$ and dimethylamine hydrochloride (DMA. HCl) $(1.2 \mathrm{~g}, 14.7 \mathrm{mmol})$ were dissolved in $40 \mathrm{~mL}$ water. Sodium molybdate dihydrate $(0.04 \mathrm{~g}, 0.16 \mathrm{mmol})$ was added to the solution. Then, $10 \mu \mathrm{L}$ of hydrazine hydrate (64-65\%) was added to the solution dropwise, followed by adjusting the $\mathrm{pH}$ to $2.40 \mathrm{by} 12 \mathrm{M}$ $\mathrm{HCl}$. The solution was heated with stirring at around $50^{\circ} \mathrm{C}$ for $30 \mathrm{mins}$. Upon cooling down to room temperature, dark blue powder formed and was removed by filtration. The dark blue precipitates appeared after a few days and were removed by filtration again. Small dark blue rod-like crystals of $\mathbf{4}$ were isolated after one month. Alternatively, sodium molybdate dihydrate and hydrazine hydrate can be replaced by molybdenum $(\mathrm{V})$ chloride $(0.1 \mathrm{~g}, 0.37 \mathrm{mmol})$ under the same conditions with $\mathrm{pH}$ ranging from 2.5 to 3.4 , which led to the formation of compound $\mathbf{4}$ as well. Note that the original synthesis of $\mathbf{4}$ involves $\left[\mathrm{Mo}_{2}{ }_{2} \mathrm{O}_{4}\right]^{2+}$ as reducing Mo species.

However, if hydrazine hydrate was not added during the synthesis, only "Trojan Horse" type clusters $\left[\mathrm{W}_{18} \mathrm{O}_{56}\left(\mathrm{HPO}_{3}\right)_{2}\left(\mathrm{H}_{2} \mathrm{O}\right)_{2}\right]^{8-[7]}$ formed as the exclusive product. Similarly, if sodium molybdate dihydrate was absent, the initially reduced solution of tungstate will be gradually oxidized during the crystallization and gave rise to "Trojan Horse" type clusters $\left[\mathrm{W}_{18} \mathrm{O}_{56}\left(\mathrm{HPO}_{3}\right)_{2}\left(\mathrm{H}_{2} \mathrm{O}\right)_{2}\right]^{8-}$ as well. Therefore, the presence of reduced Mo species is essential for the formation of compounds 1-10. The control experiments and original synthesis of $\mathbf{4}$ are summarized in Scheme $\mathbf{S} 1$ for a clearer comparison.

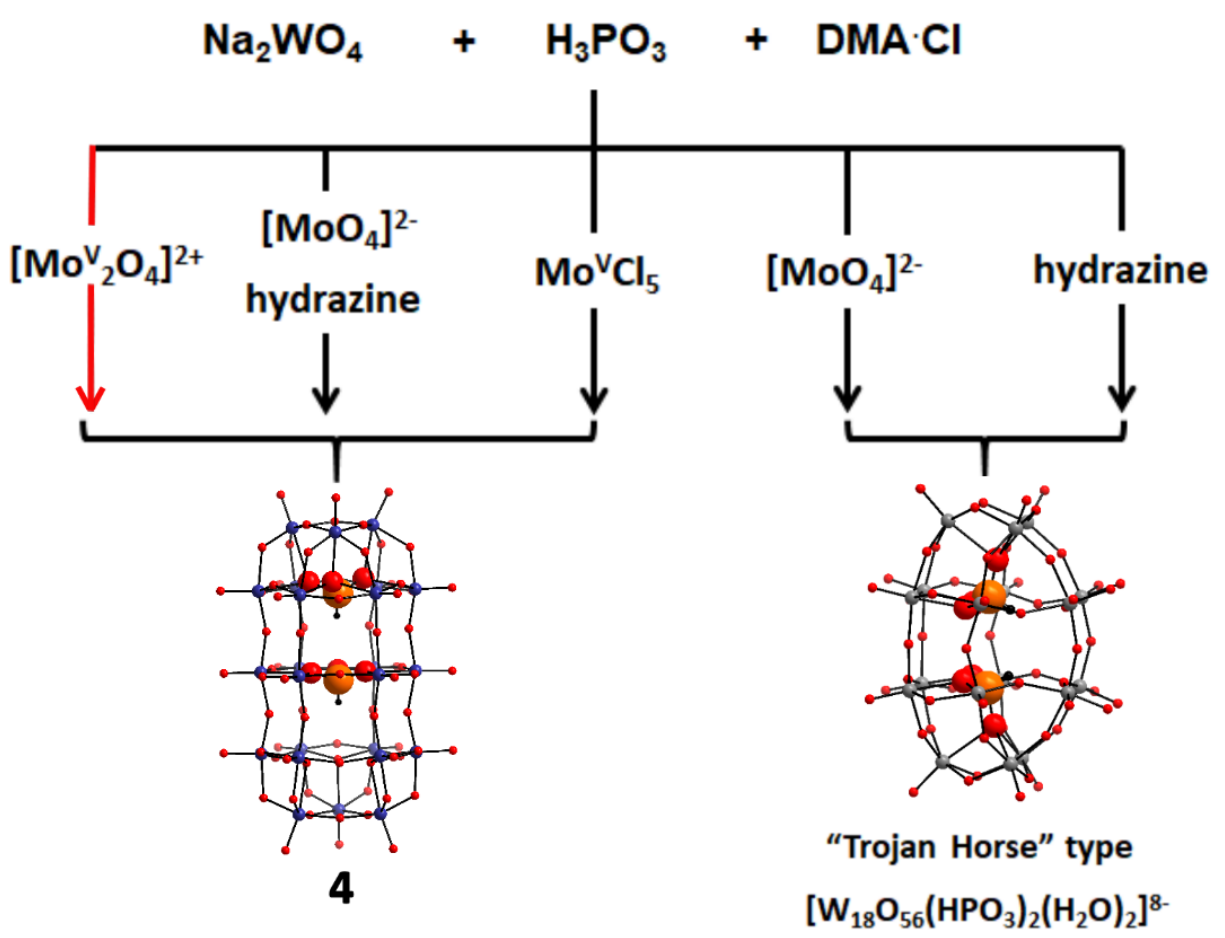

Scheme S1. Schematic representation of the original synthesis of compound 4 and control experiments, showing the key role of the reduced Mo species in directing the self-assembly of multi-layered POM clusters. The original synthesis of $\mathbf{4}$ is emphasized by the red arrow, and the control experiments are indicated by the black arrows. Colour scheme: orange: P; indigo: W/Mov; grey: W; red: O; black: hydrogen. 


\section{Single-crystal $X$-ray structure determination}

Table S1 Crystal data and structure refinement for compound 1-3.

\begin{tabular}{|c|c|c|c|}
\hline Identification code & 1 & 2 & 3 \\
\hline Empirical formula & $\mathrm{C}_{14} \mathrm{H}_{90} \mathrm{Mo}_{2} \mathrm{~N}_{7} \mathrm{O}_{75} \mathrm{PW}_{16}$ & $\mathrm{C}_{14} \mathrm{H}_{79} \mathrm{Mo}_{2} \mathrm{~N}_{7} \mathrm{O}_{70} \mathrm{SeW}_{16}$ & $\mathrm{C}_{14} \mathrm{H}_{79} \mathrm{Mo}_{2} \mathrm{~N}_{7} \mathrm{O}_{70} \mathrm{TeW}_{16}$ \\
\hline Formula weight & 4721.37 & 4678.28 & 4726.92 \\
\hline Temperature & $150(2) \mathrm{K}$ & $150(2) \mathrm{K}$ & $150(2) \mathrm{K}$ \\
\hline Wavelength & $0.71073 \AA$ & $0.71073 \AA$ & $71.073 \AA$ \\
\hline Crystal system & Trigonal & Trigonal & Trigonal \\
\hline Space group & $\mathrm{R}-3 \mathrm{~m}: \mathrm{H}$ & $\mathrm{R}-3 \mathrm{~m}: \mathrm{H}$ & $\mathrm{R}-3 \mathrm{~m}: \mathrm{H}$ \\
\hline \multirow[t]{3}{*}{ Unit cell dimensions } & $\mathrm{a}=21.5987(10) \AA$ & $\mathrm{a}=21.1690(9) \AA$ & $\mathrm{a}=21.1678(9) \AA$ \\
\hline & $\mathrm{b}=21.5987(10) \AA$ & $\mathrm{b}=21.1690(13) \AA$ & $\mathrm{b}=21.1678(9) \AA$ \\
\hline & $\mathrm{c}=13.9195(15) \AA$ & $\mathrm{c}=15.8379(12) \AA$ & $c=15.6954(14) \AA$ \\
\hline Volume & $5623.5(8) \AA^{3}$ & $6146.5(7) \AA^{3}$ & $6090.5(8) \AA^{3}$ \\
\hline $\mathrm{Z}$ & 3 & 3 & 3 \\
\hline Density (calculated) & $4.182 \mathrm{Mg} / \mathrm{m}^{3}$ & $3.792 \mathrm{Mg} / \mathrm{m}^{3}$ & $3.866 \mathrm{Mg} / \mathrm{m}^{3}$ \\
\hline Absorption coefficient & $24.903 \mathrm{~mm}^{-1}$ & $23.200 \mathrm{~mm}^{-1}$ & $23.317 \mathrm{~mm}^{-1}$ \\
\hline $\mathrm{F}(000)$ & 6318 & 6222 & 6276 \\
\hline Crystal size & $0.070 \times 0.050 \times 0.030 \mathrm{~mm}^{3}$ & $0.100 \times 0.050 \times 0.050 \mathrm{~mm}^{3}$ & $0.100 \times 0.050 \times 0.050 \mathrm{~mm}^{3}$ \\
\hline$\theta$ range for data collection & 1.824 to $26.998^{\circ}$. & 2.802 to $25.994^{\circ}$. & 2.573 to $25.723^{\circ}$. \\
\hline Index ranges & $\begin{array}{l}-15<=\mathrm{h}<=23,-15<=\mathrm{k}<=27, \\
-17<=1<=17\end{array}$ & $\begin{array}{l}-26<=\mathrm{h}<=16,-21<=\mathrm{k}<=24, \\
-19<=1<=18\end{array}$ & $\begin{array}{l}-25<=\mathrm{h}<=23,-25<=\mathrm{k}<=25, \\
-19<=\mathrm{l}<=19\end{array}$ \\
\hline Reflections collected & 6807 & 7799 & 28942 \\
\hline Independent reflections & $1450[\mathrm{R}$ (int) $=0.0807]$ & $1459[\mathrm{R}($ int $)=0.0493]$ & $1408[\mathrm{R}(\mathrm{int})=0.0553]$ \\
\hline Completeness to $\theta 25^{\circ}$ & $100.0 \%$ & $99.7 \%$ & $99.9 \%$ \\
\hline Absorption correction & Empirical & Empirical & Empirical \\
\hline Max. \& min. transmission & 0.0942 and 0.0376 & 0.093 and 0.057 & 0.547 and 0.216 \\
\hline Refinement method & $\begin{array}{l}\text { Full-matrix least-squares } \\
\text { on } \mathrm{F}^{2}\end{array}$ & $\begin{array}{l}\text { Full-matrix least-squares } \\
\text { on } \mathrm{F}^{2}\end{array}$ & $\begin{array}{l}\text { Full-matrix least-squares } \\
\text { on } \mathrm{F}^{2}\end{array}$ \\
\hline Data/restraints/parameters & $1450 / 5 / 86$ & $1459 / 4 / 100$ & $1408 / 2 / 86$ \\
\hline Goodness-of-fit on $\mathrm{F}^{2}$ & 1.118 & 1.121 & 1.237 \\
\hline Final $R$ indices $[I>2 \sigma(I)]$ & $\begin{array}{l}\mathrm{R} 1=0.0487, \mathrm{wR} 2= \\
0.1238\end{array}$ & $\begin{array}{l}\mathrm{R} 1=0.0390, \mathrm{wR} 2= \\
0.0895\end{array}$ & $\begin{array}{l}\mathrm{R} 1=0.0399, \mathrm{wR} 2= \\
0.1108\end{array}$ \\
\hline $\mathrm{R}$ indices (all data) & $\begin{array}{l}\mathrm{R} 1=0.0720, \mathrm{wR} 2= \\
0.1368\end{array}$ & $\begin{array}{l}\mathrm{R} 1=0.0563, \mathrm{wR} 2= \\
0.1010\end{array}$ & $\begin{array}{l}\mathrm{R} 1=0.0525, \mathrm{wR} 2= \\
0.1234\end{array}$ \\
\hline Extinction coefficient & $\mathrm{n} / \mathrm{a}$ & $\mathrm{n} / \mathrm{a}$ & $\mathrm{n} / \mathrm{a}$ \\
\hline $\begin{array}{l}\text { Largest diff. peak and } \\
\text { hole }\end{array}$ & 3.97 and -2.97 e. $\AA^{-3}$ & 1.07 and -2.22 e. $\AA^{-3}$ & 1.81 and -2.39 e. $\AA^{-3}$ \\
\hline
\end{tabular}


Table S2 Crystal data and structure refinement for compound 4 and 5.

\begin{tabular}{|c|c|c|}
\hline Identification code & 4 & 5 \\
\hline Empirical formula & $\mathrm{C}_{20} \mathrm{H}_{113} \mathrm{Mo}_{3} \mathrm{~N}_{10} \mathrm{Na}_{2} \mathrm{O}_{96} \mathrm{P}_{2} \mathrm{~W}_{21}$ & $\mathrm{C}_{22} \mathrm{H}_{119} \mathrm{Mo}_{3} \mathrm{~N}_{11} \mathrm{NaO}_{96} \mathrm{Se}_{2} \mathrm{~W}_{21}$ \\
\hline Formula weight & 6286.79 & 6403.85 \\
\hline Temperature & $150(2) \mathrm{K}$ & $150(2) \mathrm{K}$ \\
\hline Wavelength & $0.71073 \AA$ & $0.71073 \AA$ \\
\hline Crystal system & Orthorhombic & Orthorhombic \\
\hline Space group & $\mathrm{Pn} \mathrm{m} \mathrm{a}$ & $\mathrm{Pn} \mathrm{m} \mathrm{a}$ \\
\hline Unit cell dimensions & $\mathrm{a}=35.244(2) \AA$ & $\mathrm{a}=20.577(2) \AA$ \\
\hline & $\mathrm{b}=21.9528(13) \AA$ & $\mathrm{b}=21.446(2) \AA$ \\
\hline & $\mathrm{c}=15.0516(8) \AA$ & $\mathrm{c}=25.391(3) \AA$ \\
\hline Volume & $11645.4(11) \AA^{3}$ & $11204.7(19) \AA^{3}$ \\
\hline Z & 4 & 4 \\
\hline Density (calculated) & $3.586 \mathrm{Mg} / \mathrm{m}^{3}$ & $3.796 \mathrm{Mg} / \mathrm{m}^{3}$ \\
\hline Absorption coefficient & $21.099 \mathrm{~mm}^{-1}$ & $22.548 \mathrm{~mm}^{-1}$ \\
\hline $\mathrm{F}(000)$ & 11212 & 11420 \\
\hline Crystal size & $0.100 \times 0.060 \times 0.050 \mathrm{~mm}^{3}$ & $0.100 \times 0.050 \times 0.050 \mathrm{~mm}^{3}$ \\
\hline$\theta$ range for data collection & 1.640 to $25.999^{\circ}$. & 1.243 to $26.000^{\circ}$. \\
\hline Index ranges & $\begin{array}{l}-43<=\mathrm{h}<=43,-27<=\mathrm{k}<=25,- \\
18<=\mathrm{l}<=18\end{array}$ & $\begin{array}{l}-25<=\mathrm{h}<=25,-26<=\mathrm{k}<=26, \\
-31<=\mathrm{l}<=31\end{array}$ \\
\hline Reflections collected & 166812 & 168417 \\
\hline Independent reflections & $11754[\mathrm{R}(\mathrm{int})=0.0787]$ & $11330[\mathrm{R}(\mathrm{int})=0.0579]$ \\
\hline Completeness to $\theta 25^{\circ}$ & $99.9 \%$ & $99.9 \%$ \\
\hline Absorption correction & Empirical & Empirical \\
\hline Max. \& min. transmission & 0.0223 and 0.0091 & 0.062 and 0.012 \\
\hline Refinement method & Full-matrix least-squares on $\mathrm{F}^{2}$ & Full-matrix least-squares on $\mathrm{F}^{2}$ \\
\hline Data/restraints/parameters & 11754 / 112 / 663 & $11330 / 530 / 676$ \\
\hline Goodness-of-fit on $\mathrm{F}^{2}$ & 1.193 & 1.258 \\
\hline Final $R$ indices $[\mathrm{I}>2 \sigma(\mathrm{I})]$ & $\mathrm{R} 1=0.1167, \mathrm{wR} 2=0.2465$ & $\mathrm{R} 1=0.0754, \mathrm{wR} 2=0.1888$ \\
\hline $\mathrm{R}$ indices (all data) & $\mathrm{R} 1=0.1443, \mathrm{wR} 2=0.2791$ & $\mathrm{R} 1=0.0966, \mathrm{wR} 2=0.2179$ \\
\hline Extinction coefficient & $\mathrm{n} / \mathrm{a}$ & $\mathrm{n} / \mathrm{a}$ \\
\hline Largest diff. peak and hole & 5.59 and -9.76 e. $\AA^{-3}$ & 3.37 and -3.25 e. $\AA^{-3}$ \\
\hline
\end{tabular}


Table S3 Crystal data and structure refinement for compound 7-8.

\begin{tabular}{|c|c|c|}
\hline Identification code & 7 & 8 \\
\hline Empirical formula & $\mathrm{C}_{20} \mathrm{H}_{112} \mathrm{Mo}_{2} \mathrm{~N}_{10} \mathrm{NaO}_{96} \mathrm{PSeW}_{22}$ & $\mathrm{C}_{22} \mathrm{H}_{120} \mathrm{Mo}_{3} \mathrm{~N}_{11} \mathrm{NaO}_{96} \mathrm{PTeW}_{21}$ \\
\hline Formula weight & 6398.69 & 6405.51 \\
\hline Temperature & $150(2) \mathrm{K}$ & $150(2) \mathrm{K}$ \\
\hline Wavelength & $0.71073 \AA$ & $0.71073 \AA$ \\
\hline Crystal system & Cubic & Orthorhombic \\
\hline Space group & $\mathrm{P}$ a -3 & Pn m a \\
\hline Unit cell dimensions & $\mathrm{a}=22.5570(18) \AA$ & $\mathrm{a}=35.220(3) \AA$ \\
\hline & $\mathrm{b}=22.5570(18) \AA$ & $\mathrm{b}=22.1684(19) \AA$ \\
\hline & $\mathrm{c}=22.5570(18) \AA$ & $\mathrm{c}=15.0386(11) \AA$ \\
\hline Volume & $11477(3) \AA^{3}$ & $11741.7(16) \AA^{3}$ \\
\hline Z & 4 & 4 \\
\hline Density (calculated) & $3.703 \mathrm{Mg} / \mathrm{m}^{3}$ & $3.624 \mathrm{Mg} / \mathrm{m}^{3}$ \\
\hline Absorption coefficient & $22.599 \mathrm{~mm}^{-1}$ & $21.154 \mathrm{~mm}^{-1}$ \\
\hline $\mathrm{F}(000)$ & 11368 & 11420 \\
\hline Crystal size & $0.149 \times 0.070 \times 0.068 \mathrm{~mm}^{3}$ & $0.089 \times 0.084 \times 0.057 \mathrm{~mm}^{3}$ \\
\hline$\theta$ range for data collection & 2.019 to $25.995^{\circ}$. & 1.636 to $25.500^{\circ}$. \\
\hline Index ranges & $\begin{array}{l}-27<=\mathrm{h}<=27,-27<=\mathrm{k}<=27,- \\
27<=1<=27\end{array}$ & $-42<=\mathrm{h}<=42,-26<=\mathrm{k}<=26,-18<=\mathrm{l}<=18$ \\
\hline Reflections collected & 161282 & 166207 \\
\hline Independent reflections & $3776[\mathrm{R}(\mathrm{int})=0.0583]$ & $11238[\mathrm{R}(\mathrm{int})=0.0699]$ \\
\hline Completeness to $\theta 25^{\circ}$ & $99.9 \%$ & $99.9 \%$ \\
\hline Absorption correction & Empirical & Empirical \\
\hline Max. \& min. transmission & 0.0309 and 0.0072 & 0.0235 and 0.0083 \\
\hline Refinement method & Full-matrix least-squares on $\mathrm{F}^{2}$ & Full-matrix least-squares on $\mathrm{F}^{2}$ \\
\hline Data/restraints/parameters & $3776 / 10 / 212$ & $11238 / 510 / 703$ \\
\hline Goodness-of-fit on $\mathrm{F}^{2}$ & 1.214 & 1.203 \\
\hline Final $R$ indices $[\mathrm{I}>2 \sigma(\mathrm{I})]$ & $\mathrm{R} 1=0.0794, \mathrm{wR} 2=0.2043$ & $\mathrm{R} 1=0.1084, \mathrm{wR} 2=0.2465$ \\
\hline $\mathrm{R}$ indices (all data) & $\mathrm{R} 1=0.1004, \mathrm{wR} 2=0.2389$ & $\mathrm{R} 1=0.1200, \mathrm{wR} 2=0.2562$ \\
\hline Extinction coefficient & $\mathrm{n} / \mathrm{a}$ & $\mathrm{n} / \mathrm{a}$ \\
\hline Largest diff. peak and hole & 1.99 and -1.73 e..$\AA^{-3}$ & 4.38 and -5.98 e. $\AA^{-3}$ \\
\hline
\end{tabular}


Table S4 Crystal data and structure refinement for compound 9-11.

\begin{tabular}{|c|c|c|c|}
\hline Identification code & 9 & 10 & 11 \\
\hline Empirical formula & $\mathrm{C}_{32} \mathrm{H}_{161} \mathrm{Mo}_{4} \mathrm{~N}_{16} \mathrm{O}_{117} \mathrm{P}_{3} \mathrm{~W}_{26}$ & $\mathrm{C}_{60} \mathrm{H}_{291} \mathrm{Mo}_{4} \mathrm{~N}_{30} \mathrm{NaO}_{251} \mathrm{Se}_{7} \mathrm{~W}_{62}$ & $\mathrm{C}_{80} \mathrm{H}_{183} \mathrm{Mo}_{2} \mathrm{~N}_{5} \mathrm{O}_{60} \mathrm{TeW}_{16}$ \\
\hline Formula weight & 7899.53 & 17808.38 & 5436.38 \\
\hline Temperature & $150(2) \mathrm{K}$ & $150(2) \mathrm{K}$ & $150(2) \mathrm{K}$ \\
\hline Wavelength & $0.71073 \AA$ & $0.71073 \AA$ & $0.71073 \AA$ \\
\hline Crystal system & Orthorhombic & Triclinic & Trigonal \\
\hline Space group & $\mathrm{C} \mathrm{m} \mathrm{c} \mathrm{a}$ & $\mathrm{P}-1$ & $\mathrm{R}-3: \mathrm{H}$ \\
\hline \multirow[t]{6}{*}{ Unit cell dimensions } & $\mathrm{a}=21.7592(18) \AA$ & $\mathrm{a}=17.7735(13) \AA$ & $\mathrm{a}=44.713(3) \AA$ \\
\hline & $\mathrm{b}=26.906(2) \AA$ & $\mathrm{b}=20.1721(14) \AA$ & $\mathrm{b}=44.713(3) \AA$ \\
\hline & $\mathrm{c}=25.728(2) \AA$ & $c=22.3319(16) \AA$ & $\mathrm{c}=47.217(5) \AA$ \\
\hline & & $\alpha=99.133(4)^{\circ}$. & \\
\hline & & $\beta=102.654(4)^{\circ}$. & \\
\hline & & $\gamma=96.166(4)^{\circ}$. & \\
\hline Volume & $15062(2) \AA^{3}$ & $7628.6(10) \AA^{3}$ & $81751(14) \AA^{3}$ \\
\hline Z & 4 & 1 & 24 \\
\hline Density (calculated) & $3.484 \mathrm{Mg} / \mathrm{m}^{3}$ & $3.876 \mathrm{Mg} / \mathrm{m}^{3}$ & $2.650 \mathrm{Mg} / \mathrm{m}^{3}$ \\
\hline Absorption coefficient & $20.221 \mathrm{~mm}^{-1}$ & $24.365 \mathrm{~mm}^{-1}$ & $13.911 \mathrm{~mm}^{-1}$ \\
\hline $\mathrm{F}(000)$ & 14152 & 7874 & 59952 \\
\hline Crystal size & $\begin{array}{l}0.100 \times 0.100 \times 0.100 \\
\mathrm{~mm}^{3}\end{array}$ & $0.100 \times 0.050 \times 0.050 \mathrm{~mm}^{3}$ & $\begin{array}{l}0.100 \times 0.100 \times 0.060 \\
\mathrm{~mm}^{3}\end{array}$ \\
\hline$\theta$ range for data collection & 1.872 to $26.449^{\circ}$. & 1.455 to $26.000^{\circ}$. & 0.680 to $23.298^{\circ}$. \\
\hline Index ranges & $\begin{array}{l}-25<=\mathrm{h}<=27,- \\
33<=\mathrm{k}<=30, \\
-30<=\mathrm{k}<=32\end{array}$ & $\begin{array}{l}-21<=\mathrm{h}<=19,-24<=\mathrm{k}<=24, \\
-27<=\mathrm{l}<=26\end{array}$ & $\begin{array}{l}-49<=\mathrm{h}<=46,- \\
49<=\mathrm{k}<=49,-52<=\mathrm{l}<=52\end{array}$ \\
\hline Reflections collected & 61753 & 115963 & 122591 \\
\hline Independent reflections & $7946[\mathrm{R}(\mathrm{int})=0.0429]$ & $29949[\mathrm{R}(\mathrm{int})=0.0503]$ & $23878[\mathrm{R}(\mathrm{int})=0.0818]$ \\
\hline Completeness to $\theta 25^{\circ}$ & $99.9 \%$ & $99.9 \%$ & $91.0 \%$ \\
\hline Absorption correction & Empirical & Analytical & Empirical \\
\hline Max. \& min. transmission & 0.0085 and 0.0020 & 0.0526 and 0.0122 & 0.0886 and 0.0378 \\
\hline Refinement method & $\begin{array}{l}\text { Full-matrix least-squares } \\
\text { on } \mathrm{F}^{2}\end{array}$ & $\begin{array}{l}\text { Full-matrix least-squares on } \\
\mathrm{F}^{2}\end{array}$ & $\begin{array}{l}\text { Full-matrix least-squares } \\
\text { on } \mathrm{F}^{2}\end{array}$ \\
\hline Data/restraints/parameters & 7946 / 30 / 439 & $29949 / 72$ / 1634 & $23878 / 43 / 1149$ \\
\hline Goodness-of-fit on $\mathrm{F}^{2}$ & 1.184 & 1.095 & 1.075 \\
\hline Final $R$ indices $[\mathrm{I}>2 \sigma(\mathrm{I})]$ & $\begin{array}{l}\mathrm{R} 1=0.0514, \mathrm{wR} 2= \\
0.1393\end{array}$ & $\mathrm{R} 1=0.0569, \mathrm{wR} 2=0.1465$ & $\begin{array}{l}\mathrm{R} 1=0.0772, \mathrm{wR} 2= \\
0.2088\end{array}$ \\
\hline $\mathrm{R}$ indices (all data) & $\begin{array}{l}\mathrm{R} 1=0.0766, \mathrm{wR} 2= \\
0.1804\end{array}$ & $\mathrm{R} 1=0.0903, \mathrm{wR} 2=0.1755$ & $\begin{array}{l}\mathrm{R} 1=0.1430, \mathrm{wR} 2= \\
0.2747\end{array}$ \\
\hline Extinction coefficient & $\mathrm{n} / \mathrm{a}$ & $\mathrm{n} / \mathrm{a}$ & $\mathrm{n} / \mathrm{a}$ \\
\hline
\end{tabular}




\begin{tabular}{|l|l|l|l|}
\hline $\begin{array}{l}\text { Largest diff. peak and } \\
\text { hole }\end{array}$ & 2.27 and $-2.18 \mathrm{e} . \AA^{-3}$ & 3.73 and $-5.32 \mathrm{e} . \AA^{-3}$ & 2.49 and $-1.71 \mathrm{e} . \AA^{-3}$ \\
\hline
\end{tabular}

\section{Structure solution and refinement details}

All structures were solved by using direct method and refined by full-matrix least-squares on F2. All W/Mo sites were initially refined as $\mathrm{W}$ with free occupancies. Then a ratio of W/Mo occupancy was calculated and fixed for each site in further refinements using EXYZ and EADP instructions. The sum of W or Mo contents are considered and adjusted by referring to chemical analysis results. Hetero atoms $\mathrm{P}$, Se and Te were identified by checking their X-O bond distances. Their occupancies were initially freely refined and then fixed. Disorders of hetero atoms were modeled by considering their orientations. Because the templates are all trigonal hetero anions. The lone pair (or hydrogen in the phosphorus case) repulsion requires two heteroatoms separate in a considerable distance when they are in face-to-face orientations. The heteroatom on the layer near cluster end can only orientate towards the cluster center, otherwise the lone pair would interact with the cluster end capping $\{\mathrm{W} 3\}$ triangle. So, if all the layers are occupied by heteroatoms, there will be occurrence that two heteroatoms orientate in face-to-face mode and the heteroatoms locate too close. Therefore, there will be one layer is unoccupied in the case of Se and Te and almost in the case of $\mathrm{P}$, whose atomic size is smaller. For the two-layer structures 1-3, one hetero atom is disordered over two positions with half occupancy on each position. In compound 1, the phosphorus case, there is a very little portion that maybe both positions are occupied simultaneously. Structure refinement found the sum of occupancies of the two positions are slightly higher than 1. Compound 4 ideally has two phosphorus hetero atoms locating over three positions with occupancy of 0.666 for each position, see Figure S1. However, in real structure refinement of 4 , the sum of occupancies of the three positions are slightly higher than 2 , like the phosphorus case in compound 1, and the occupancies for three positions are slightly unequal but all close to 0.666 . Also, it was observed that there is a little chance for compound 1 to co-crystallize in compound $\mathbf{4}$ as shown by the two additional tungsten positions W14 and W15 in the structure of $\mathbf{4}$. Compound $\mathbf{6}$, the bullet structure, has the up two layers mainly ( $90 \%$ ) occupied by Se atoms and the bottom layer is nearly empty (only around $10 \%$ occupancy). This shows either the Se atom occupies the bottom layer with very low occupancy or the bullet cluster turnovers in molecule packing with small chance. In compound $\mathbf{7}$ and $\mathbf{8}$ of the mix heteroatoms, $\mathrm{P}$ was always found located in the middle layer with full occupancies while Se or Te on the two side layers. Locations and occupancies are shown in Figure S2. In compound 9, it was found that there is only one arrangement for three phosphorus atoms on four positions and two molecule packing mode. Occupancies found in structure refinement for each site are shown in Figure S3. In $\left[\mathrm{W}_{26} \mathrm{Mo}_{4} \mathrm{O}_{93}\left(\mathrm{SeO}_{3}\right)_{3}\right]^{16-}\left(\mathbf{1 0}^{10 a}\right)$, three selenium atoms are located on four heteroatom positions and the average occupancy is 0.75 for each layer as illustrated in Figure S4 as found in structure refinement.

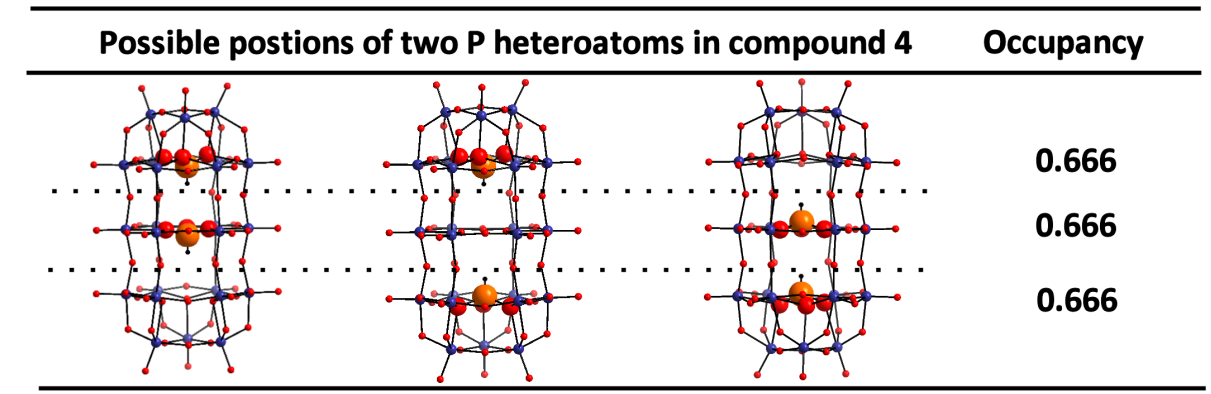

Figure S1. Two phosphorus atoms are disordered over three heteroatom positions in compound $\mathbf{4}$, with an average occupancy of 0.666 on each position.

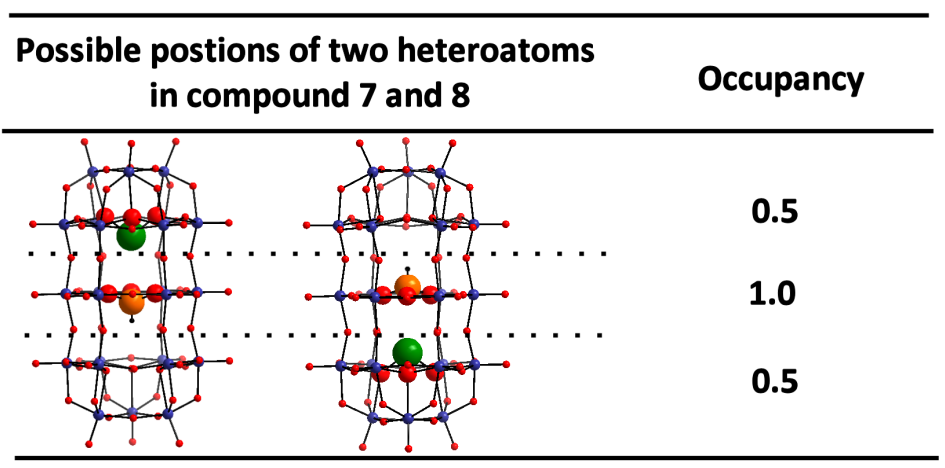

Figure S2. Selenium or tellurium atoms (green) are disordered equally at the two ends, whereas phosphorus (orange) fully occupy the middle-layer position in compound $\mathbf{7}$ and $\mathbf{8}$. 


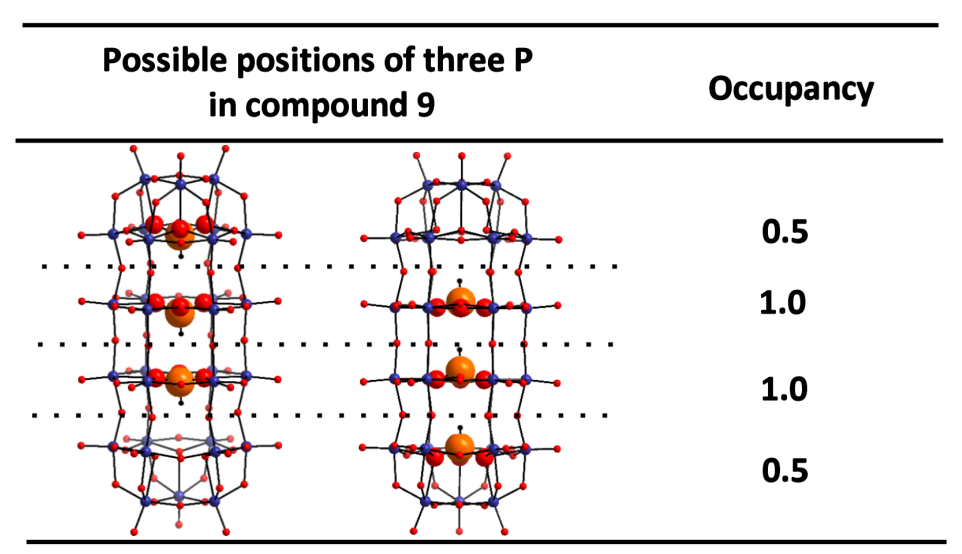

Figure S3. One phosphorus atom is disordered equally at the two end layers, and other two phosphorus atoms fully occupy the two middle-layer positions in compound $\mathbf{9}$.

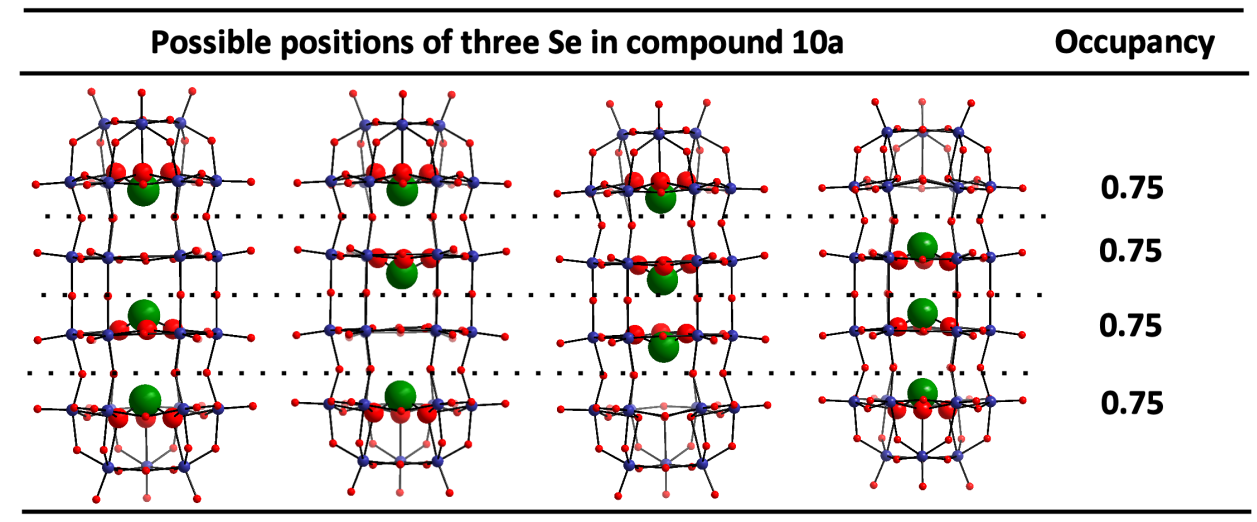

Figure S4. Three selenium atoms are disordered over four heteroatom positions in cluster 10a, with an average occupancy of 0.75 on each position.

\section{UV-Vis-NIR spectroscopy}

(a)

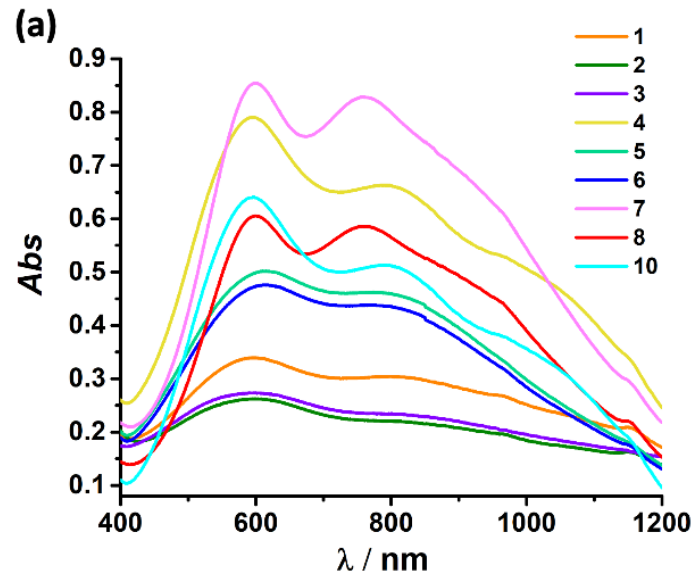

(b)

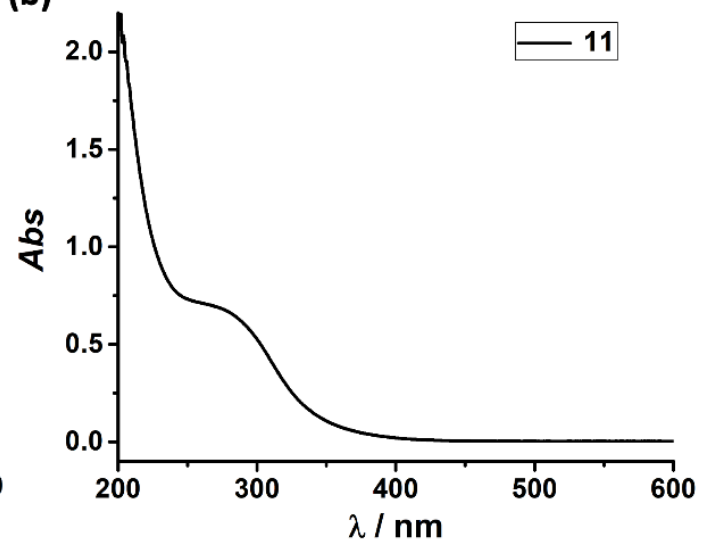

Figure S5. UV-vis-NIR spectroscopy of a) compound 1-8, 10 and b) compound 11. 


\section{ESI-mass spectrometry}

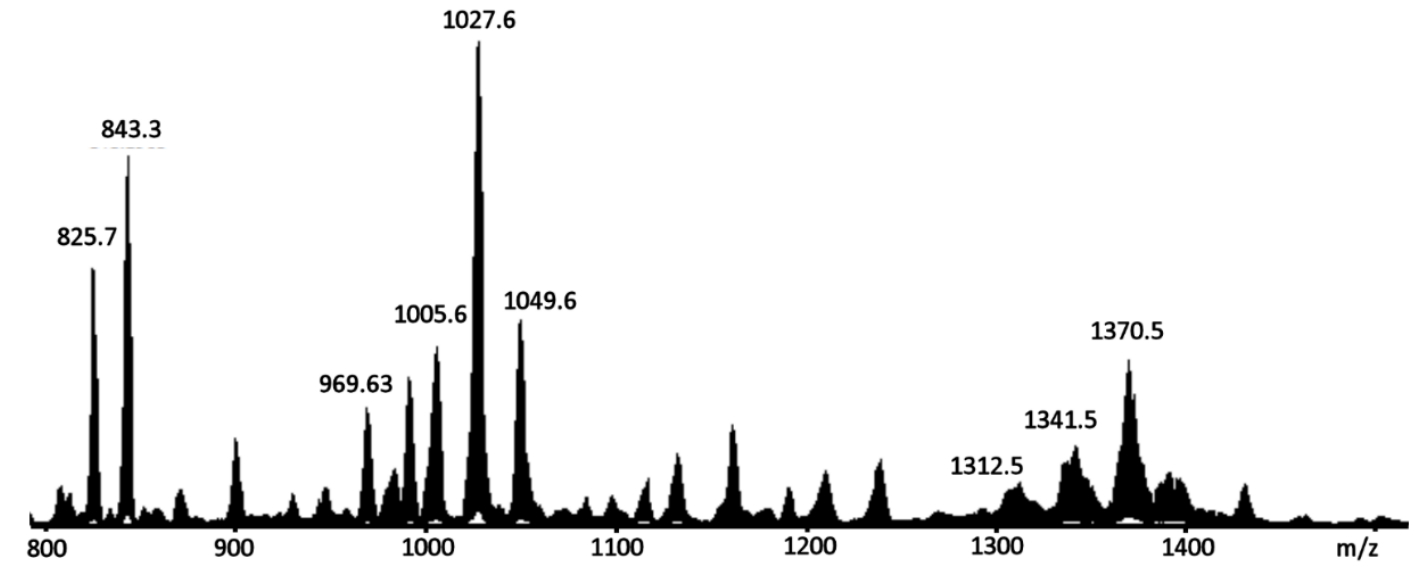

Figure S6. ESI-MS spectra of 1 in water/acetonitrile (5\%:95 \%) mixture solvent.

Table S5. Mass spectral analysis of 1 in water/acetonitrile (5\%:95\%) mixture solvent, with proposed formula and charge of peaks.

\begin{tabular}{|c|c|c|c|}
\hline z & $\mathrm{m} / \mathrm{z}$ (obs) & $\mathrm{m} / \mathrm{z}$ (cal) & Assignment \\
\hline \multirow{2}{*}{$5-$} & 825.7 & 825.6 & $\mathrm{HPW}_{16} \mathrm{Mo}_{2} \mathrm{O}_{60} \mathrm{H}_{3}$ \\
\hline & 843.3 & 843.2 & $\mathrm{HPW}_{17} \mathrm{Mo}_{1} \mathrm{O}_{60} \mathrm{H}_{3}$ \\
\hline \multirow{5}{*}{ 4- } & 983.6 & 983.2 & $\mathrm{HPW}_{13} \mathrm{Mo}_{5} \mathrm{O}_{60}\left(\mathrm{C}_{2} \mathrm{H}_{8} \mathrm{~N}\right)_{1} \mathrm{Na}_{1} \mathrm{H}_{2}$ \\
\hline & 991.6 & 992.2 & $\mathrm{HPW}_{13} \mathrm{Mo}_{5} \mathrm{O}_{60}\left(\mathrm{C}_{2} \mathrm{H}_{8} \mathrm{~N}\right)_{1} \mathrm{Na}_{1} \mathrm{H}_{2}\left(\mathrm{H}_{2} \mathrm{O}\right)$ \\
\hline & 1005.6 & 1005.2 & $\mathrm{HPW}_{14} \mathrm{Mo}_{4} \mathrm{O}_{60}\left(\mathrm{C}_{2} \mathrm{H}_{8} \mathrm{~N}\right)_{1} \mathrm{Na}_{1} \mathrm{H}_{2}$ \\
\hline & 1027.6 & 1027.2 & $\mathrm{HPW}_{15} \mathrm{Mo}_{3} \mathrm{O}_{60}\left(\mathrm{C}_{2} \mathrm{H}_{8} \mathrm{~N}\right)_{1} \mathrm{Na}_{1} \mathrm{H}_{2}$ \\
\hline & 1049.6 & 1048.9 & $\mathrm{HPW}_{16} \mathrm{Mo}_{2} \mathrm{O}_{60}\left(\mathrm{C}_{2} \mathrm{H}_{8} \mathrm{~N}\right)_{1} \mathrm{Na}_{1} \mathrm{H}_{2}$ \\
\hline \multirow{2}{*}{$3-$} & 1312.5 & 1311.9 & $\mathrm{HPW}_{12} \mathrm{Mo}_{6} \mathrm{O}_{60}\left(\mathrm{C}_{2} \mathrm{H}_{8} \mathrm{~N}\right)_{3} \mathrm{Na}_{1} \mathrm{H}_{1}$ \\
\hline & 1370.5 & 1370.6 & $\mathrm{HPW}_{14} \mathrm{Mo}_{4} \mathrm{O}_{60}\left(\mathrm{C}_{2} \mathrm{H}_{8} \mathrm{~N}\right)_{3} \mathrm{Na}_{1} \mathrm{H}_{1}$ \\
\hline
\end{tabular}

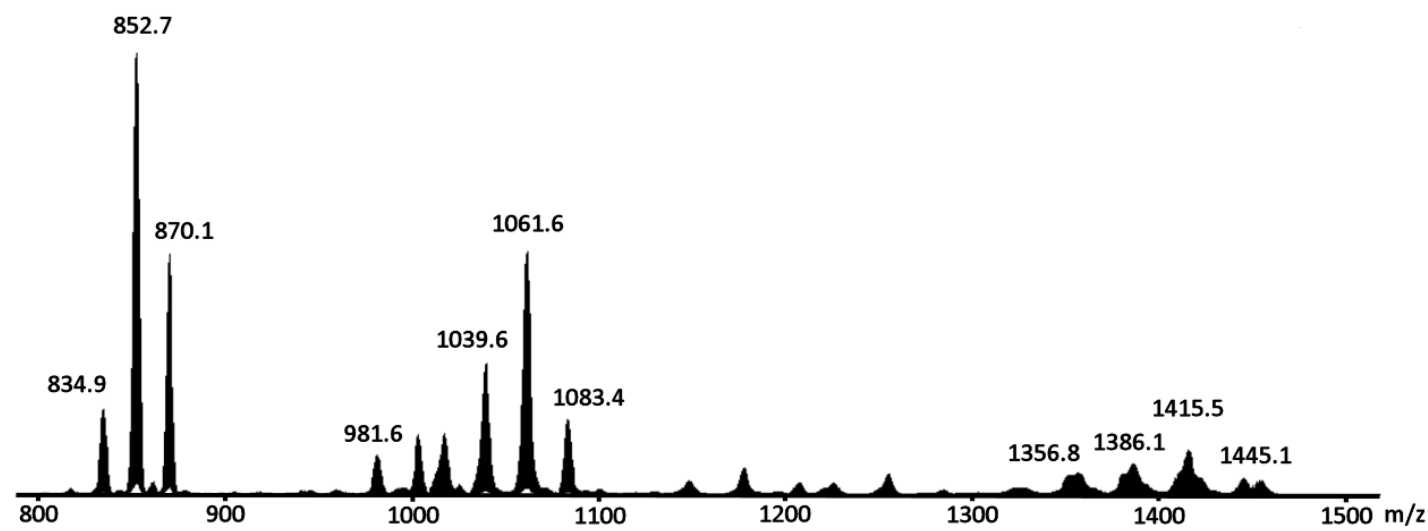

Figure S7. ESI-MS spectra of 2 in water/acetonitrile (5\%:95\%) mixture solvent.

Table S6. Mass spectral analysis of 2 in water/acetonitrile (5\%:95 \%) mixture solvent, with proposed formula and charge of peaks

\begin{tabular}{|c|c|c|c|}
\hline$z$ & $\mathrm{~m} / \mathrm{z}$ (obs) & $\mathrm{m} / \mathrm{z}$ (cal) & Assignment \\
\hline 5- & $\begin{array}{l}834.9 \\
852.7 \\
870.1 \\
\end{array}$ & $\begin{array}{l}835.1 \\
853.1 \\
869.4 \\
\end{array}$ & $\begin{array}{l}\mathrm{SeW}_{16} \mathrm{Mo}_{2} \mathrm{O}_{60} \mathrm{H}_{3} \\
\mathrm{SeW}_{16} \mathrm{Mo}_{2} \mathrm{O}_{60}\left(\mathrm{C}_{2} \mathrm{H}_{8} \mathrm{~N}\right)_{2} \mathrm{H}_{1} \\
\mathrm{SeW}_{16} \mathrm{Mo}_{2} \mathrm{O}_{60}\left(\mathrm{C}_{2} \mathrm{H}_{8} \mathrm{~N}\right)_{3}\left(\mathrm{H}_{2} \mathrm{O}\right)_{2}\end{array}$ \\
\hline 4- & $\begin{array}{c}981.6 \\
1003.6 \\
1017.6 \\
1039.6 \\
1061.6 \\
1083.4 \\
\end{array}$ & $\begin{array}{c}982.6 \\
1004.6 \\
1016.9 \\
1038.9 \\
1060.9 \\
1083.5\end{array}$ & $\begin{array}{l}\mathrm{SeW}_{13} \mathrm{Mo}_{5} \mathrm{O}_{60} \mathrm{H}_{4}\left(\mathrm{H}_{2} \mathrm{O}\right)_{1} \\
\mathrm{SeW}_{13} \mathrm{Mo}_{5} \mathrm{O}_{60} \mathrm{Na}_{4}\left(\mathrm{H}_{2} \mathrm{O}\right)_{1} \\
\mathrm{SeW}_{14} \mathrm{Mo}_{4} \mathrm{O}_{60}\left(\mathrm{C}_{2} \mathrm{H}_{8} \mathrm{~N}\right)_{1} \mathrm{Na}_{1} \mathrm{H}_{2} \\
\mathrm{SeW}_{15} \mathrm{Mo}_{3} \mathrm{O}_{60}\left(\mathrm{C}_{2} \mathrm{H}_{8} \mathrm{~N}\right)_{1} \mathrm{Na}_{1} \mathrm{H}_{2} \\
\mathrm{SeW}_{16} \mathrm{Mo}_{2} \mathrm{O}_{60}\left(\mathrm{C}_{2} \mathrm{H}_{8} \mathrm{~N}\right)_{1} \mathrm{Na}_{1} \mathrm{H}_{2} \\
\mathrm{SeW}_{16} \mathrm{Mo}_{2} \mathrm{O}_{60}\left(\mathrm{C}_{2} \mathrm{H}_{8} \mathrm{~N}\right)_{3} \mathrm{Na}_{1}\end{array}$ \\
\hline 3- & $\begin{array}{l}1303.5 \\
1322.4 \\
1327.4 \\
1352.1 \\
1356.8 \\
1381.8 \\
1386.1\end{array}$ & $\begin{array}{l}1304.5 \\
1323.8 \\
1326.5 \\
1353.2 \\
1356.2 \\
1382.5 \\
1385.6\end{array}$ & $\begin{array}{l}\mathrm{SeW}_{13} \mathrm{Mo}_{5} \mathrm{O}_{60} \mathrm{H}_{5} \\
\mathrm{SeW}_{13} \mathrm{Mo}_{5} \mathrm{O}_{60} \mathrm{Na}_{1} \mathrm{H}_{4}\left(\mathrm{H}_{2} \mathrm{O}\right)_{2} \\
\mathrm{SeW}_{13} \mathrm{Mo}_{5} \mathrm{O}_{60} \mathrm{Na}_{3} \mathrm{H}_{2} \\
\mathrm{SeW}_{14} \mathrm{Mo}_{4} \mathrm{O}_{60} \mathrm{Na}_{4} \mathrm{H}_{1}\left(\mathrm{H}_{2} \mathrm{O}\right)_{2} \\
\mathrm{SeW}_{14} \mathrm{Mo}_{4} \mathrm{O}_{60}\left(\mathrm{C}_{2} \mathrm{H}_{8} \mathrm{~N}\right)_{1} \mathrm{Na}_{1} \mathrm{H}_{3} \\
\mathrm{SeW}_{15} \mathrm{Mo}_{3} \mathrm{O}_{60}\left(\mathrm{C}_{2} \mathrm{H}_{8} \mathrm{~N}\right)_{1} \mathrm{Na}_{1} \mathrm{H}_{4}\left(\mathrm{H}_{2} \mathrm{O}\right)_{2} \\
\mathrm{SeW}_{15} \mathrm{Mo}_{3} \mathrm{O}_{60}\left(\mathrm{C}_{2} \mathrm{H}_{8} \mathrm{~N}\right)_{1} \mathrm{Na}_{1} \mathrm{H}_{3}\end{array}$ \\
\hline
\end{tabular}




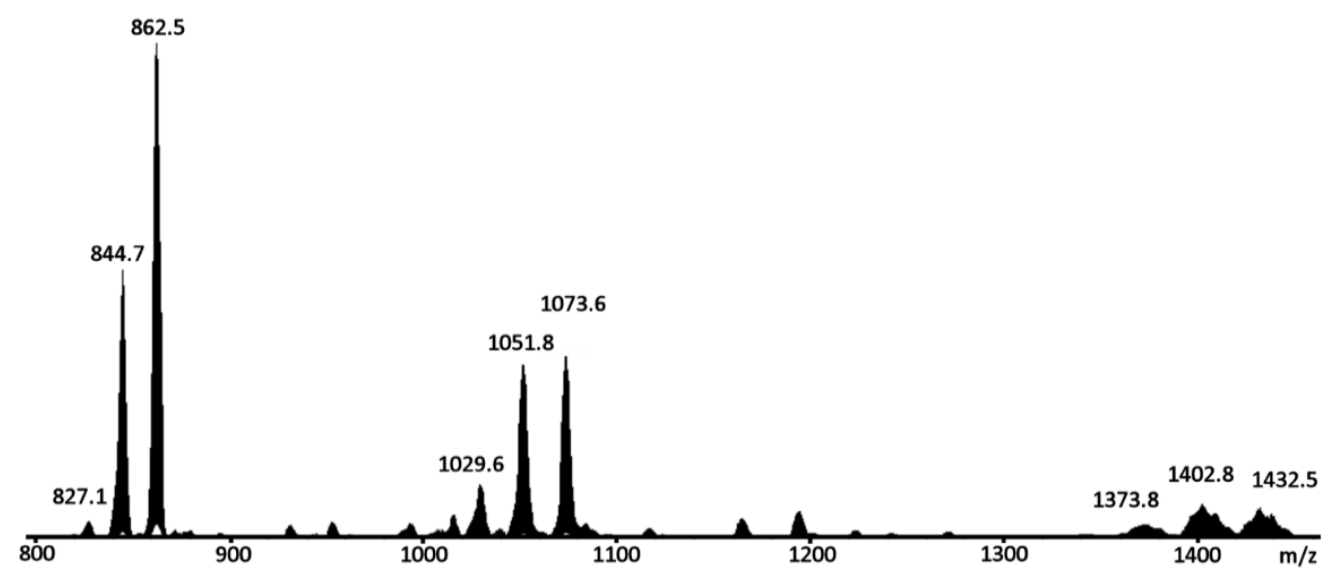

Figure S8. ESI-MS spectra of 3 in water/acetonitrile (5\%:95\%) mixture solvent.

Table S7. Mass spectral analysis of 3 in water/acetonitrile (5\%:95\%) mixture solvent, with proposed formula and charge of peaks

\begin{tabular}{cccl}
\hline$z$ & $\mathrm{~m} / \mathrm{z}$ (obs) & $\mathrm{m} / \mathrm{z}$ (cal) & Assignment \\
\hline \multirow{3}{*}{$5-$} & 827.1 & 827.1 & $\mathrm{TeW}_{15} \mathrm{Mo}_{3} \mathrm{O}_{60} \mathrm{H}_{3}$ \\
& 844.7 & 845.1 & $\mathrm{TeW}_{15} \mathrm{Mo}_{3} \mathrm{O}_{60}\left(\mathrm{C}_{2} \mathrm{H}_{8} \mathrm{~N}\right)_{2} \mathrm{H}_{1}$ \\
& 862.5 & 862.7 & $\mathrm{TeW}_{16} \mathrm{Mo}_{2} \mathrm{O}_{60}\left(\mathrm{C}_{2} \mathrm{H}_{8} \mathrm{~N}\right)_{2} \mathrm{H}_{1}$ \\
\hline \multirow{3}{*}{$4-$} & 993.6 & 994.9 & $\mathrm{TeW}_{13} \mathrm{Mo}_{5} \mathrm{O}_{60} \mathrm{H}_{4}\left(\mathrm{H}_{2} \mathrm{O}\right)$ \\
& 1015.4 & 1016.9 & $\mathrm{TeW}_{14} \mathrm{Mo}_{4} \mathrm{O}_{60} \mathrm{H}_{4}\left(\mathrm{H}_{2} \mathrm{O}\right)$ \\
& 1029.6 & 1029.1 & $\mathrm{TeW}_{14} \mathrm{Mo}_{4} \mathrm{O}_{60}\left(\mathrm{C}_{2} \mathrm{H}_{8} \mathrm{~N}\right)_{1} \mathrm{Na}_{1} \mathrm{H}_{2}$ \\
& 1051.8 & 1051.7 & $\mathrm{TeW}_{14} \mathrm{Mo}_{4} \mathrm{O}_{60}\left(\mathrm{C}_{2} \mathrm{H}_{8} \mathrm{~N}\right)_{3} \mathrm{Na}_{1}$ \\
& 1073.6 & 1073.4 & $\mathrm{TeW}_{14} \mathrm{Mo}_{4} \mathrm{O}_{60}\left(\mathrm{C}_{2} \mathrm{H}_{8} \mathrm{~N}\right)_{3} \mathrm{Na}_{1}$ \\
\hline & 1362.1 & 1363.5 & $\mathrm{TeW}_{14} \mathrm{Mo}_{4} \mathrm{O}_{60} \mathrm{Na}_{1} \mathrm{H}_{4}\left(\mathrm{H}_{2} \mathrm{O}\right)_{1}$ \\
& 1373.8 & 1372.6 & $\mathrm{TeW}_{14} \mathrm{Mo}_{4} \mathrm{O}_{60}\left(\mathrm{C}_{2} \mathrm{H}_{8} \mathrm{~N}\right)_{1} \mathrm{Na}_{1} \mathrm{H}_{3}$ \\
& 1379.4 & 1379.2 & $\mathrm{TeW}_{14} \mathrm{Mo}_{4} \mathrm{O}_{60} \mathrm{H}_{5}$ \\
& 1397.4 & 1398.5 & $\mathrm{TeW}_{15} \mathrm{Mo}_{3} \mathrm{O}_{60} \mathrm{Na}_{1} \mathrm{H}_{4}\left(\mathrm{H}_{2} \mathrm{O}\right)_{2}$ \\
& 1402.8 & 1401.5 & $\mathrm{TeW}_{15} \mathrm{Mo}_{3} \mathrm{O}_{60}\left(\mathrm{C}_{2} \mathrm{H}_{8} \mathrm{~N}\right)_{1} \mathrm{Na}_{1} \mathrm{H}_{3}$ \\
& 1409.1 & 1409.2 & $\mathrm{TeW}_{15} \mathrm{Mo}_{3} \mathrm{O}_{60}\left(\mathrm{C}_{2} \mathrm{H}_{8} \mathrm{~N}\right)_{2} \mathrm{H}_{3}$ \\
& 1427.4 & 1427.9 & $\mathrm{TeW}_{16} \mathrm{Mo}_{2} \mathrm{O}_{60} \mathrm{Na}_{1} \mathrm{H}_{4}\left(\mathrm{H}_{2} \mathrm{O}\right)_{2}$ \\
& 1432.5 & 1432.6 & $\mathrm{TeW}_{16} \mathrm{Mo}_{2} \mathrm{O}_{60} \mathrm{H}_{5}\left(\mathrm{H}_{2} \mathrm{O}\right)_{4}$ \\
& 1438.5 & 1438.6 & $\mathrm{TeW}_{16} \mathrm{Mo}_{2} \mathrm{O}_{60}\left(\mathrm{C}_{2} \mathrm{H}_{8} \mathrm{~N}\right)_{2} \mathrm{H}_{3}$ \\
& 1444.8 & 1445.9 & $\mathrm{TeW}_{16} \mathrm{Mo}_{2} \mathrm{O}_{60}\left(\mathrm{C}_{2} \mathrm{H}_{8} \mathrm{~N}\right)_{2} \mathrm{Na}_{1} \mathrm{H}_{2}$ \\
\hline
\end{tabular}

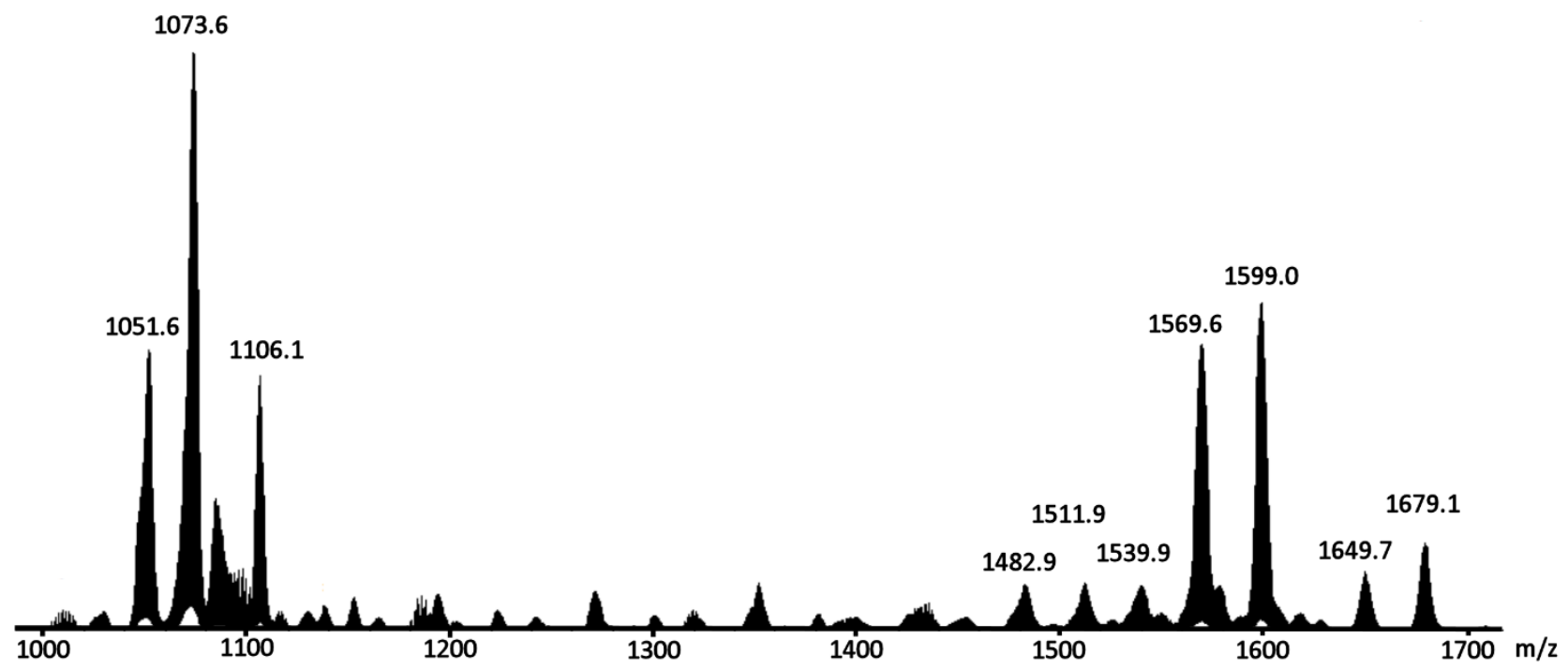

Figure S9. ESI-MS spectra of 11 in acetonitrile. 
Table S8. Mass spectral analysis of 11 in acetonitrile with proposed formula and charge of peaks

\begin{tabular}{|c|c|c|c|}
\hline$z$ & $\mathrm{~m} / \mathrm{z}$ (obs) & $\mathrm{m} / \mathrm{z}$ (cal) & Assignment \\
\hline \multirow{7}{*}{-3} & 1679.0 & 1679.5 & $\left(\mathrm{TeW}_{17} \mathrm{Mo}_{1} \mathrm{O}_{60}\right)\left(\mathrm{C}_{16} \mathrm{H}_{36} \mathrm{~N}\right)_{3} \mathrm{H}_{2}$ \\
\hline & 1649.7 & 1650.2 & $\left(\mathrm{TeW}_{16} \mathrm{Mo}_{2} \mathrm{O}_{60}\right)\left(\mathrm{C}_{16} \mathrm{H}_{36} \mathrm{~N}\right)_{3} \mathrm{H}_{2}$ \\
\hline & 1599.0 & 1598.7 & $\left(\mathrm{TeW}_{17} \mathrm{Mo}_{1} \mathrm{O}_{60}\right)\left(\mathrm{C}_{16} \mathrm{H}_{36} \mathrm{~N}\right)_{2} \mathrm{H}_{3}$ \\
\hline & 1570.0 & 1569.4 & $\left(\mathrm{TeW}_{16} \mathrm{Mo}_{2} \mathrm{O}_{60}\right)\left(\mathrm{C}_{16} \mathrm{H}_{36} \mathrm{~N}\right)_{2} \mathrm{H}_{3}$ \\
\hline & 1539.9 & 1540.4 & $\left(\mathrm{TeW}_{15} \mathrm{Mo}_{3} \mathrm{O}_{60}\right)\left(\mathrm{C}_{16} \mathrm{H}_{36} \mathrm{~N}\right)_{2} \mathrm{H}_{3}$ \\
\hline & 1511.9 & 1511.0 & $\left(\mathrm{TeW}_{14} \mathrm{Mo}_{4} \mathrm{O}_{60}\right)\left(\mathrm{C}_{16} \mathrm{H}_{36} \mathrm{~N}\right)_{2} \mathrm{H}_{3}$ \\
\hline & 1482.9 & 1481.7 & $\left(\mathrm{TeW}_{13} \mathrm{Mo}_{5} \mathrm{O}_{60}\right)\left(\mathrm{C}_{16} \mathrm{H}_{36} \mathrm{~N}\right)_{2} \mathrm{H}_{3}$ \\
\hline \multirow{5}{*}{-4} & 1138.4 & 1138.5 & $\left(\mathrm{TeW}_{17} \mathrm{Mo}_{1} \mathrm{O}_{60}\right)\left(\mathrm{C}_{16} \mathrm{H}_{36} \mathrm{~N}\right)_{1} \mathrm{H}_{3}$ \\
\hline & 1106.1 & 1107.4 & $\left(\mathrm{TeW}_{16} \mathrm{Mo}_{2} \mathrm{O}_{60}\right)\left(\mathrm{CH}_{3} \mathrm{CN}\right)_{5} \mathrm{H}_{4}$ \\
\hline & 1084.9 & 1085.7 & $\left(\mathrm{TeW}_{15} \mathrm{Mo}_{3} \mathrm{O}_{60}\right)\left(\mathrm{CH}_{3} \mathrm{CN}\right)_{5} \mathrm{H}_{4}$ \\
\hline & 1073.6 & 1072.7 & $\left(\mathrm{TeW}_{14} \mathrm{Mo}_{4} \mathrm{O}_{60}\right)\left(\mathrm{C}_{16} \mathrm{H}_{36} \mathrm{~N}\right)_{1} \mathrm{H}_{3}$ \\
\hline & 1051.3 & 1051.0 & $\left(\mathrm{TeW}_{13} \mathrm{Mo}_{5} \mathrm{O}_{60}\right)\left(\mathrm{C}_{16} \mathrm{H}_{36} \mathrm{~N}\right)_{1} \mathrm{H}_{3}$ \\
\hline
\end{tabular}

\section{NMR study}

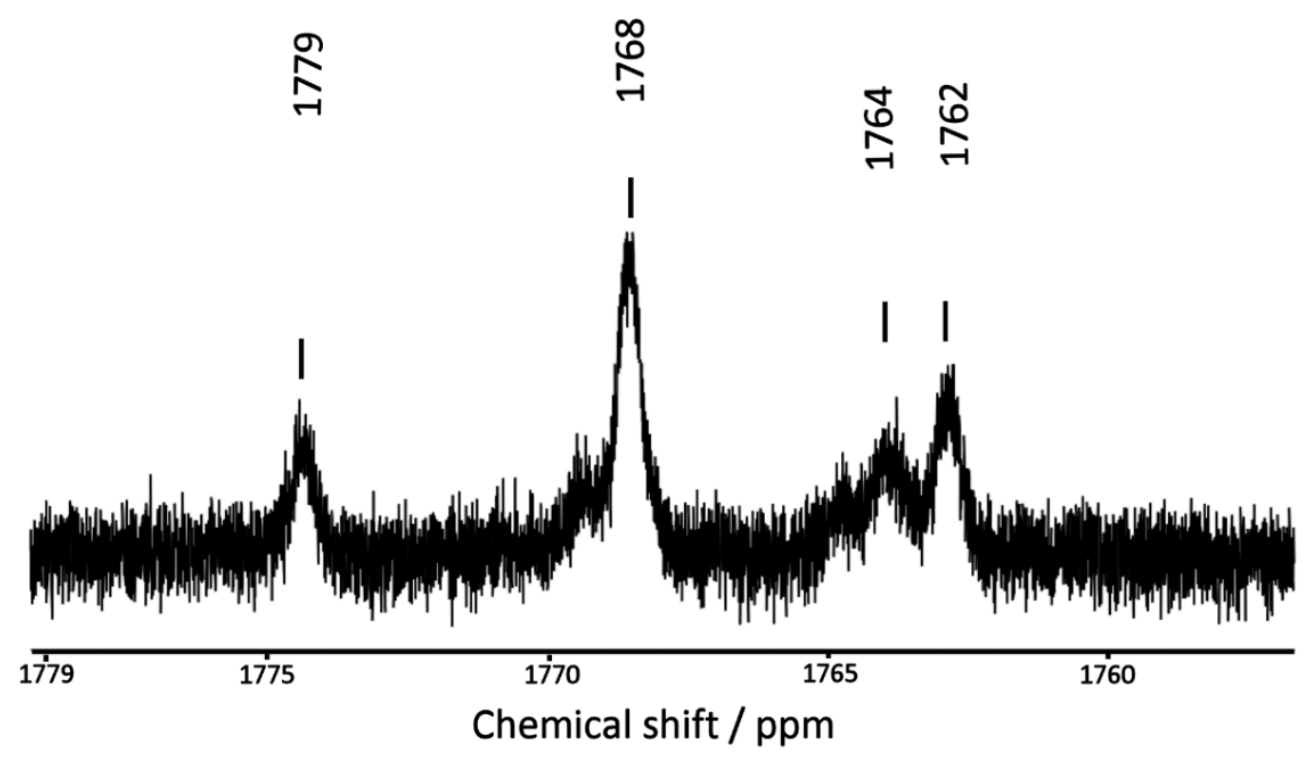

Figure S10. ${ }^{125} \mathrm{Te}$ NMR spectrum of $\mathbf{1 1}$ in dimethyl sulfoxide- $\mathrm{d}_{6}$. 


\section{Dielectric properties}

Figure S11. Temperature dependence of real $\varepsilon_{1}(a$ and $b)$ and imaginary $\varepsilon_{2}$ (c and d) parts of the complex dielectric constant for 4 , characterized by the impedance method in the frequency range from $100 \mathrm{~Hz}$ to $1 \mathrm{MHz}$ and were plotted.

(a)

(c)
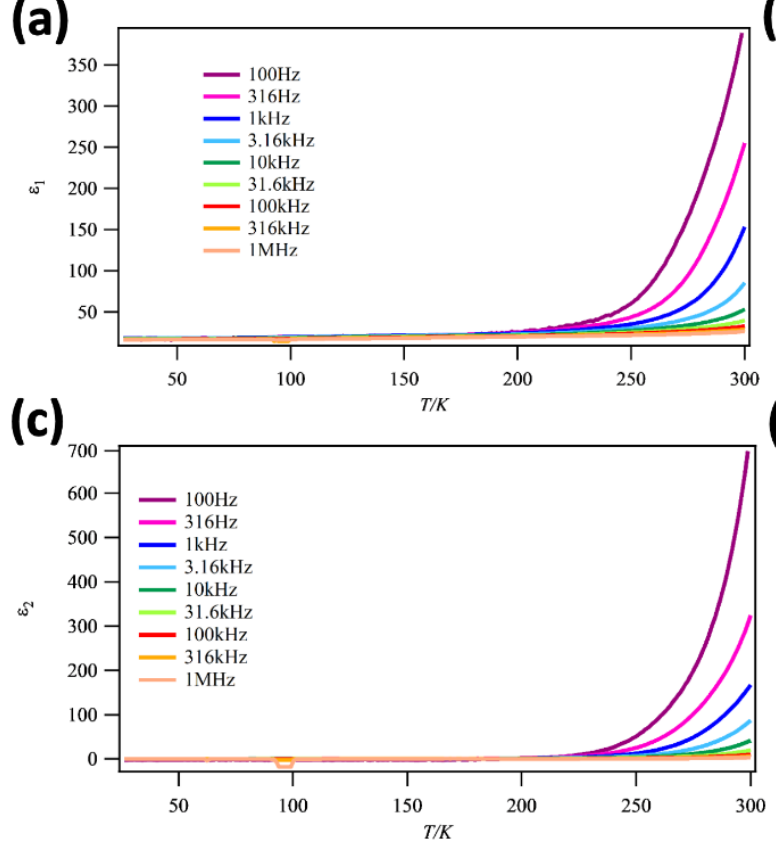

(b)

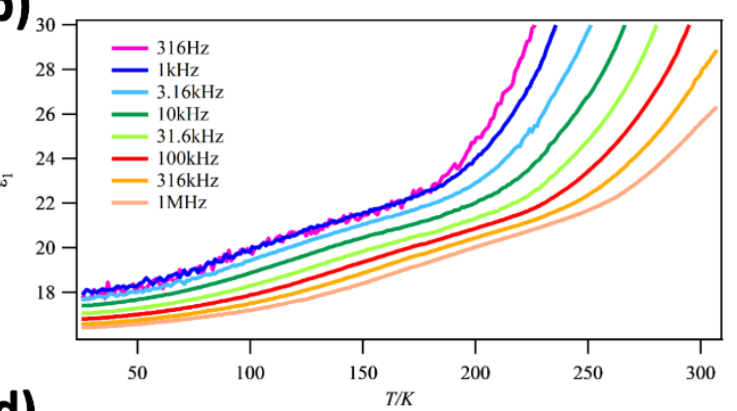

(d)

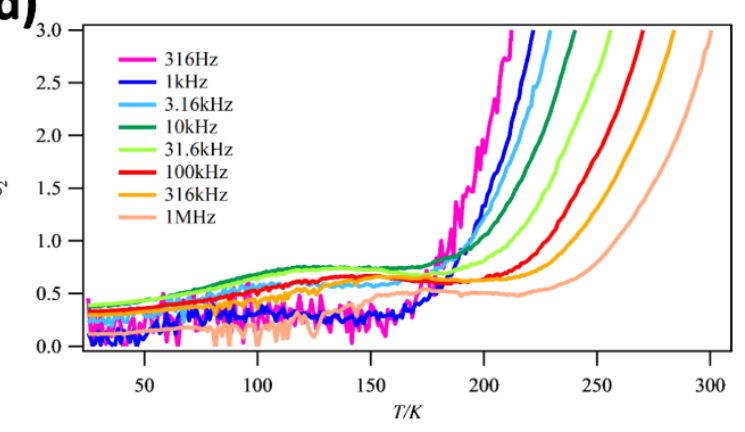

(a)

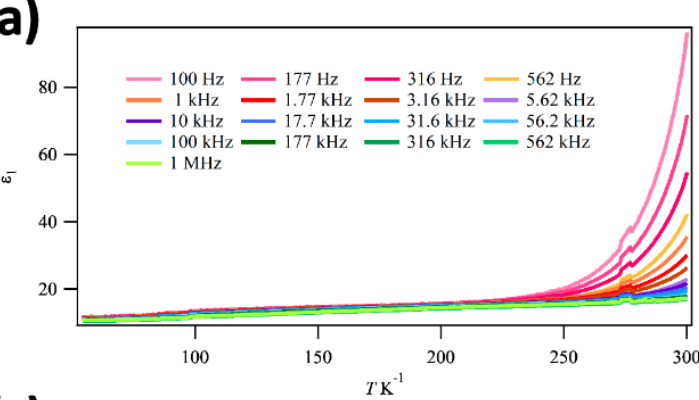

(c)

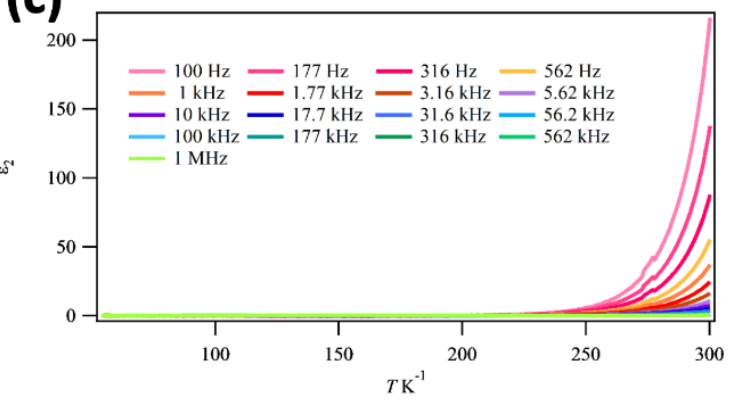

(b)

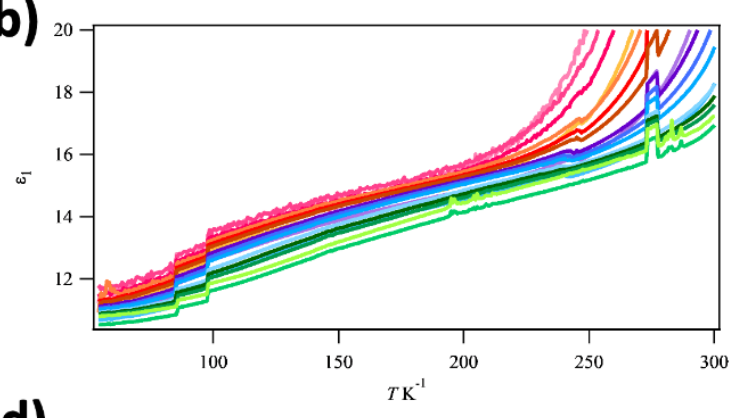

(d)

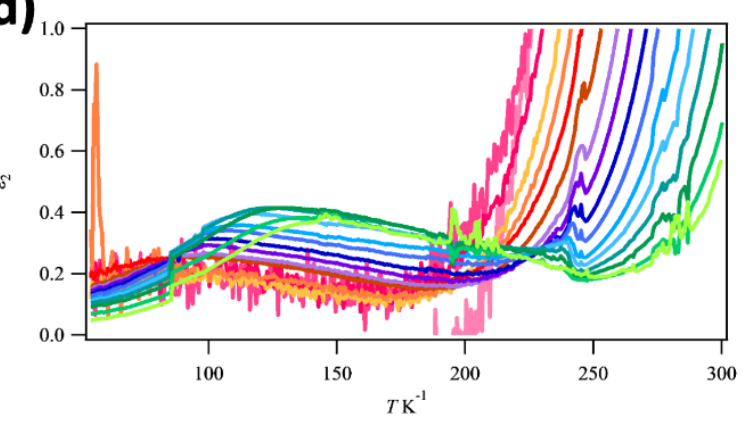

Figure S12. Temperature dependence of real $\varepsilon_{1}(a$ and $b)$ and imaginary $\varepsilon_{2}$ (c and d) parts of the complex dielectric constant for $\mathbf{6}$, characterized by the impedance method in the frequency range from $100 \mathrm{~Hz}$ to $1 \mathrm{MHz}$ and were plotted. 


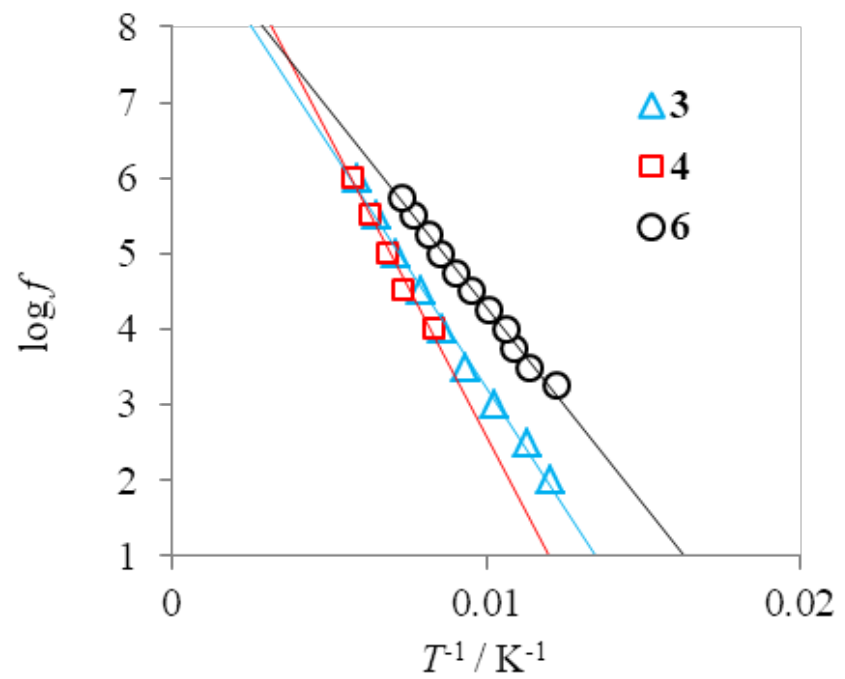

Figure S13. Arrhenius plots of compound 3, $\mathbf{4}$ and $\mathbf{6}$. Inverse of peak temperatures were plotted with logarithm of measurement frequency $f$.

Table S9. Summary of peak temperatures of $\varepsilon_{2}$ (compound $\mathbf{3}$ ).

\begin{tabular}{cc}
$\boldsymbol{f} / \mathbf{H z}$ & Peak temperature / K \\
\hline 100 & 83 \\
316 & 88 \\
1000 & 98 \\
3160 & 107 \\
10000 & 116 \\
31600 & 126 \\
100000 & 140 \\
316000 & 154 \\
1000000 & 170
\end{tabular}

Table S10. Summary of peak temperatures of $\varepsilon_{2}$ (compound 4 ).

\begin{tabular}{cc}
$\boldsymbol{f} / \mathbf{H z}$ & Peak temperature / K \\
\hline 10000 & 120 \\
31600 & 136 \\
100000 & 145 \\
316000 & 158 \\
1000000 & 174
\end{tabular}

Table S11. Summary of peak temperatures of $\varepsilon_{2}$ (compound 6).

\begin{tabular}{cc}
$\boldsymbol{f} / \mathbf{H z}$ & Peak temperature $/ \mathbf{K}$ \\
\hline 1770 & 82 \\
3160 & 88 \\
5620 & 92 \\
10000 & 94 \\
17700 & 99 \\
31600 & 105 \\
56200 & 111 \\
100000 & 117 \\
177000 & 122 \\
316000 & 130 \\
562000 & 137
\end{tabular}


8. TGA analysis

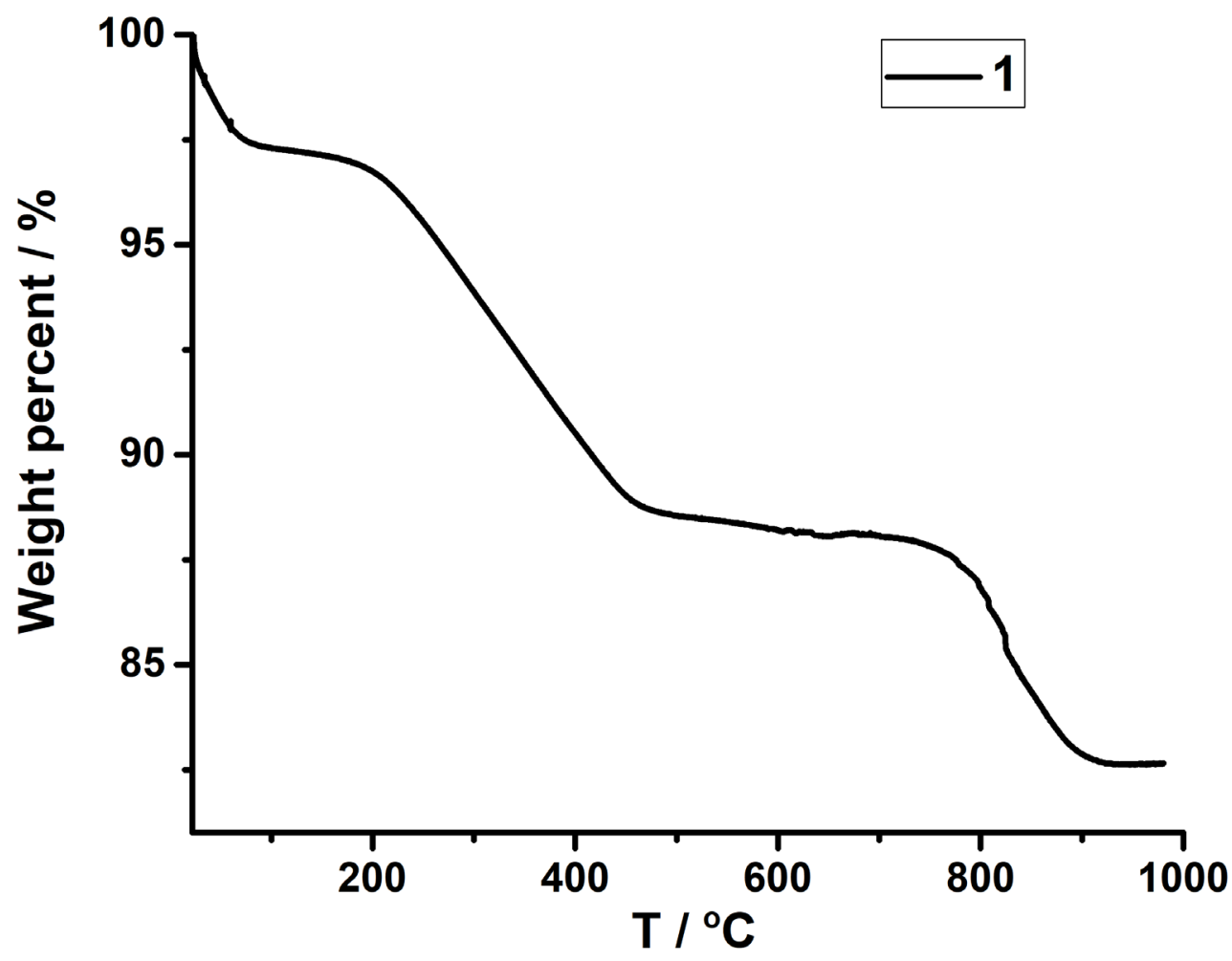

Figure S14. TGA curve for compound 1.

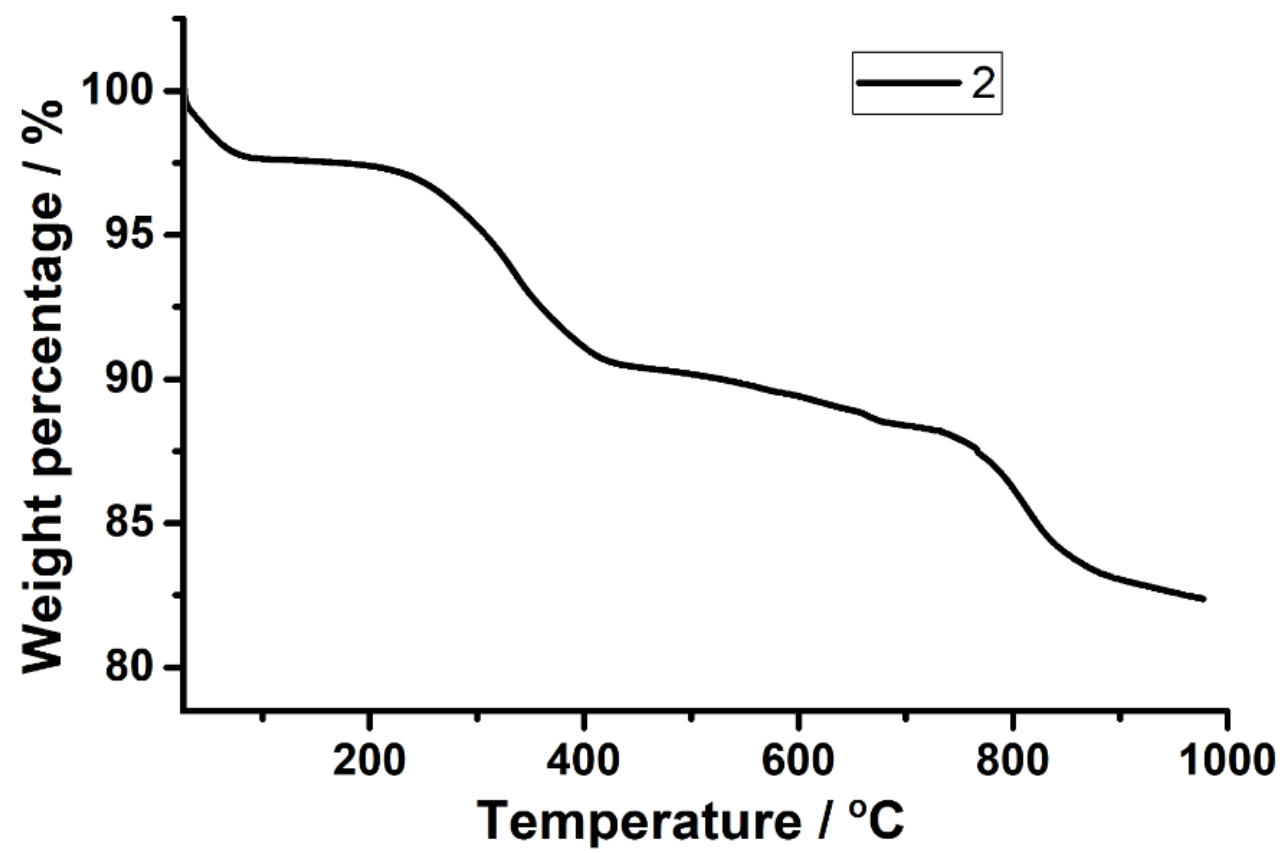

Figure S15. TGA curve for compound 2. 


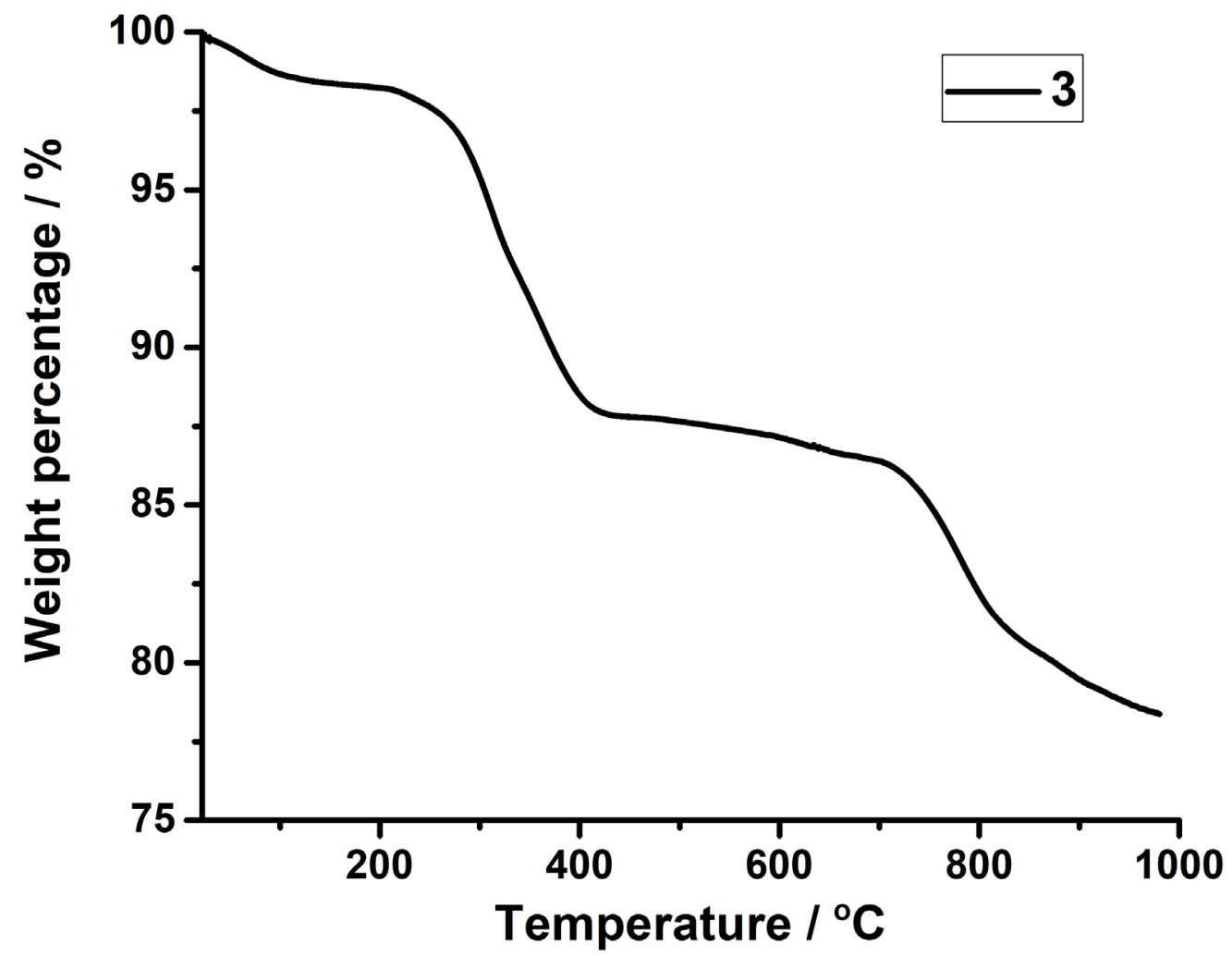

Figure S16. TGA curve for compound 3.

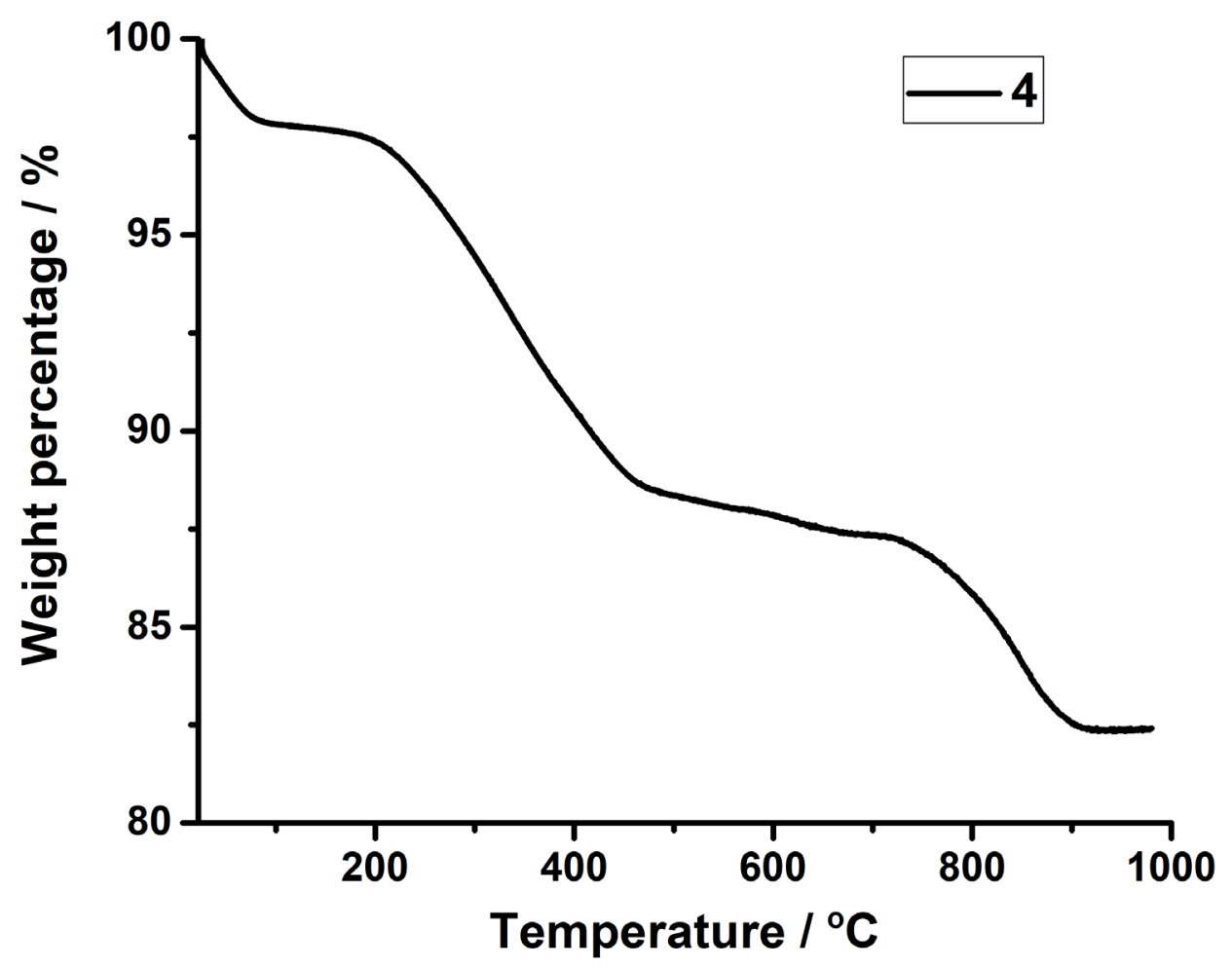

Figure S17. TGA curve for compound 4 


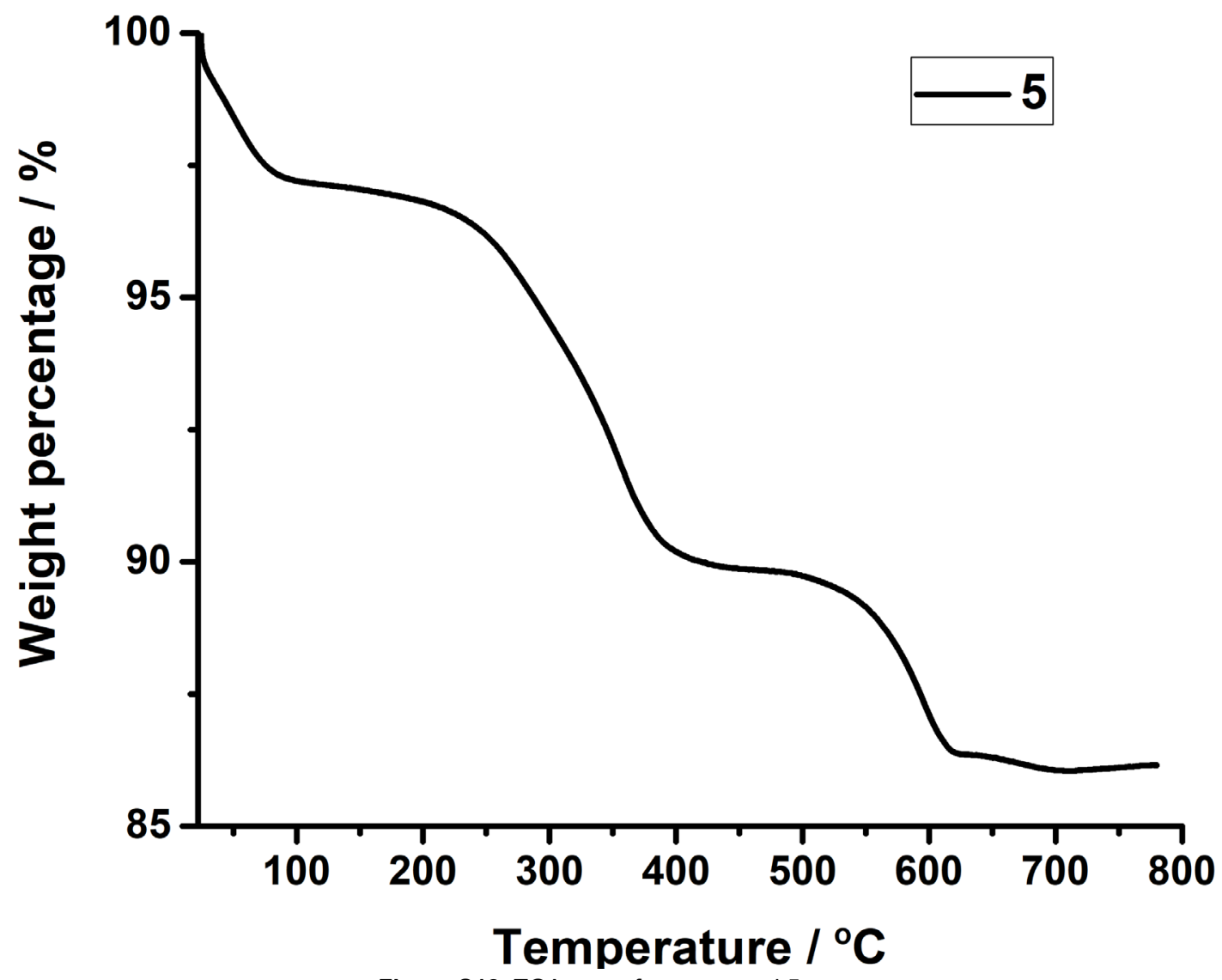

Figure S18. TGA curve for compound 5 .

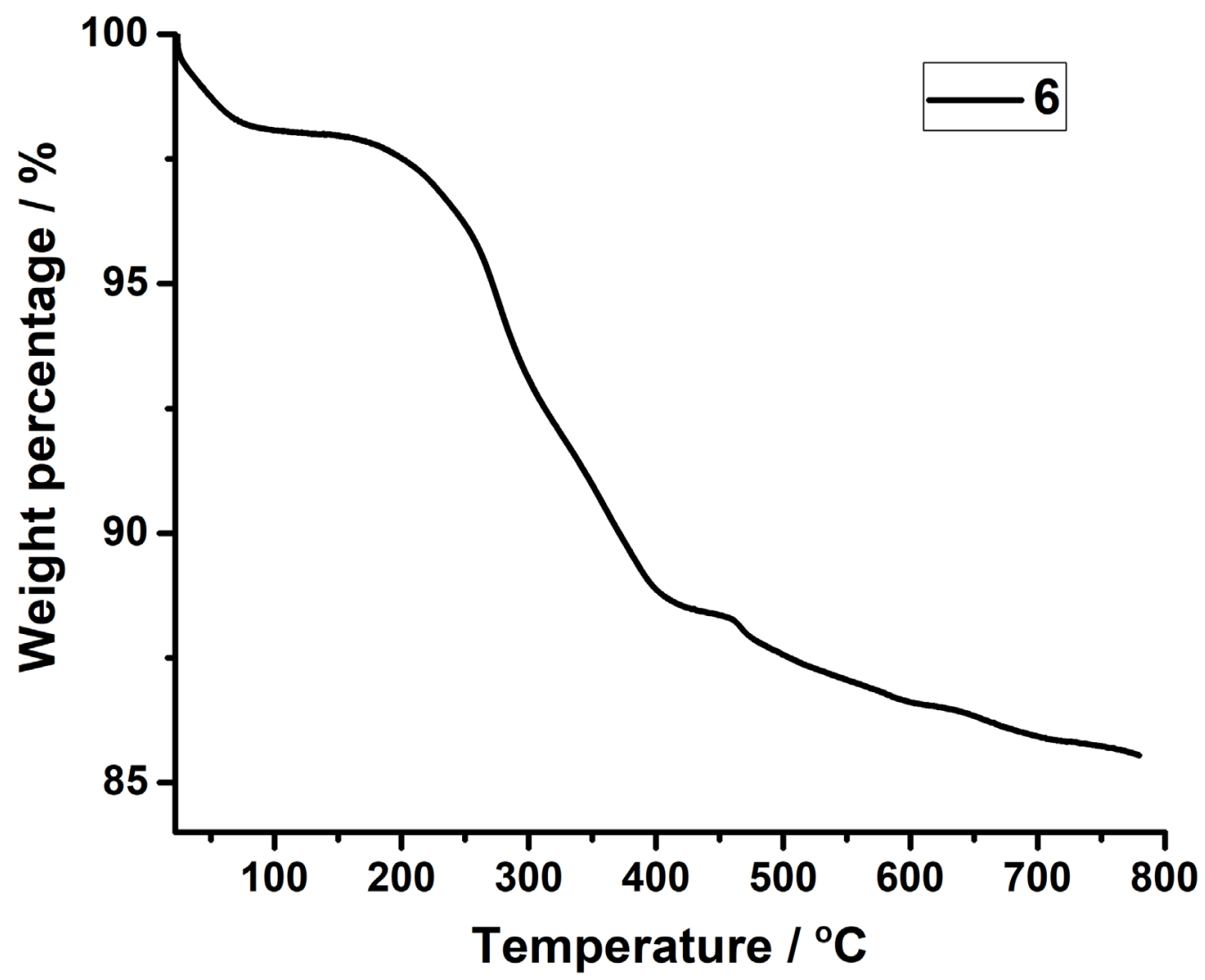

Figure S19. TGA curve for compound 6.

S19 


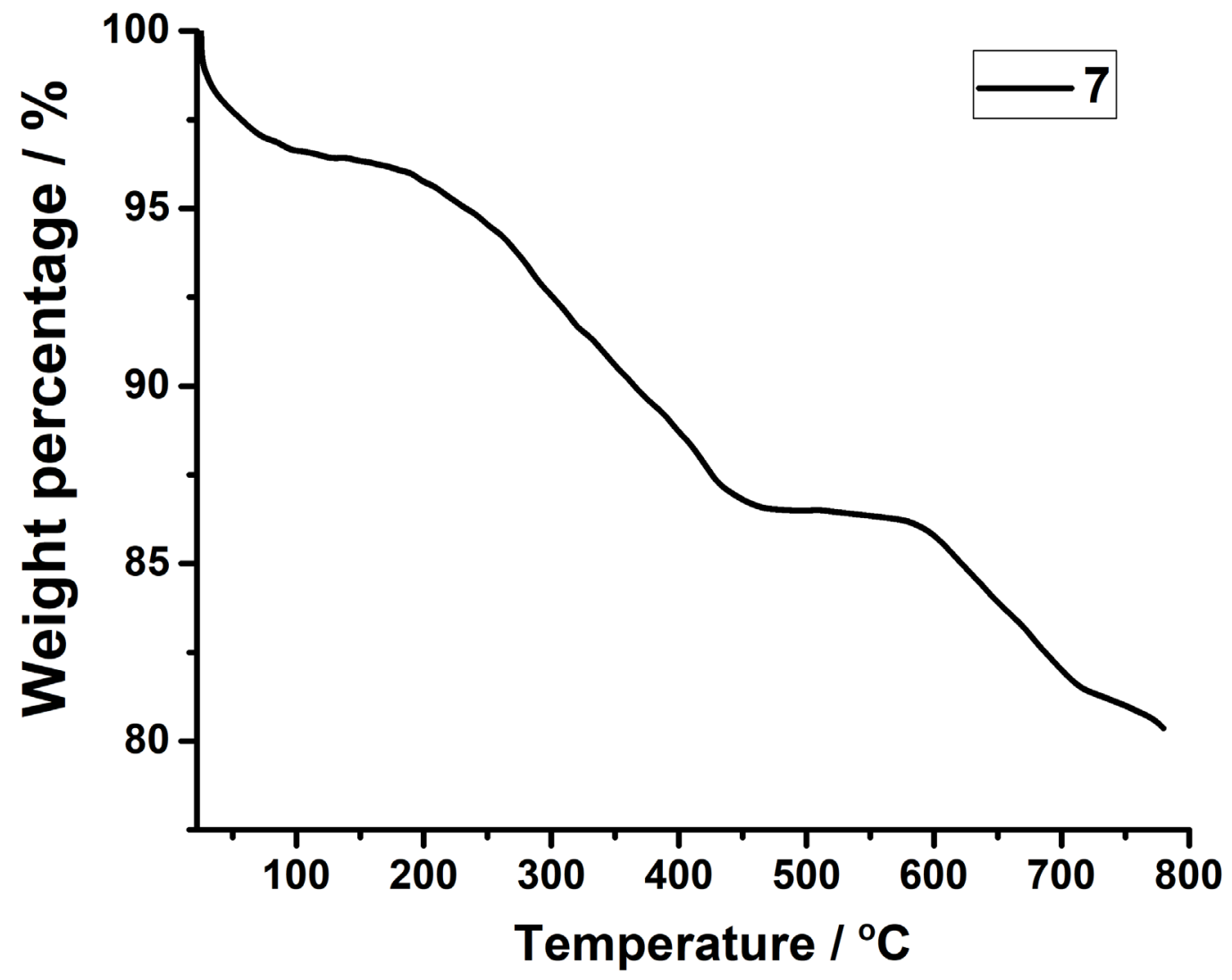

Figure S20. TGA curve for compound 7.

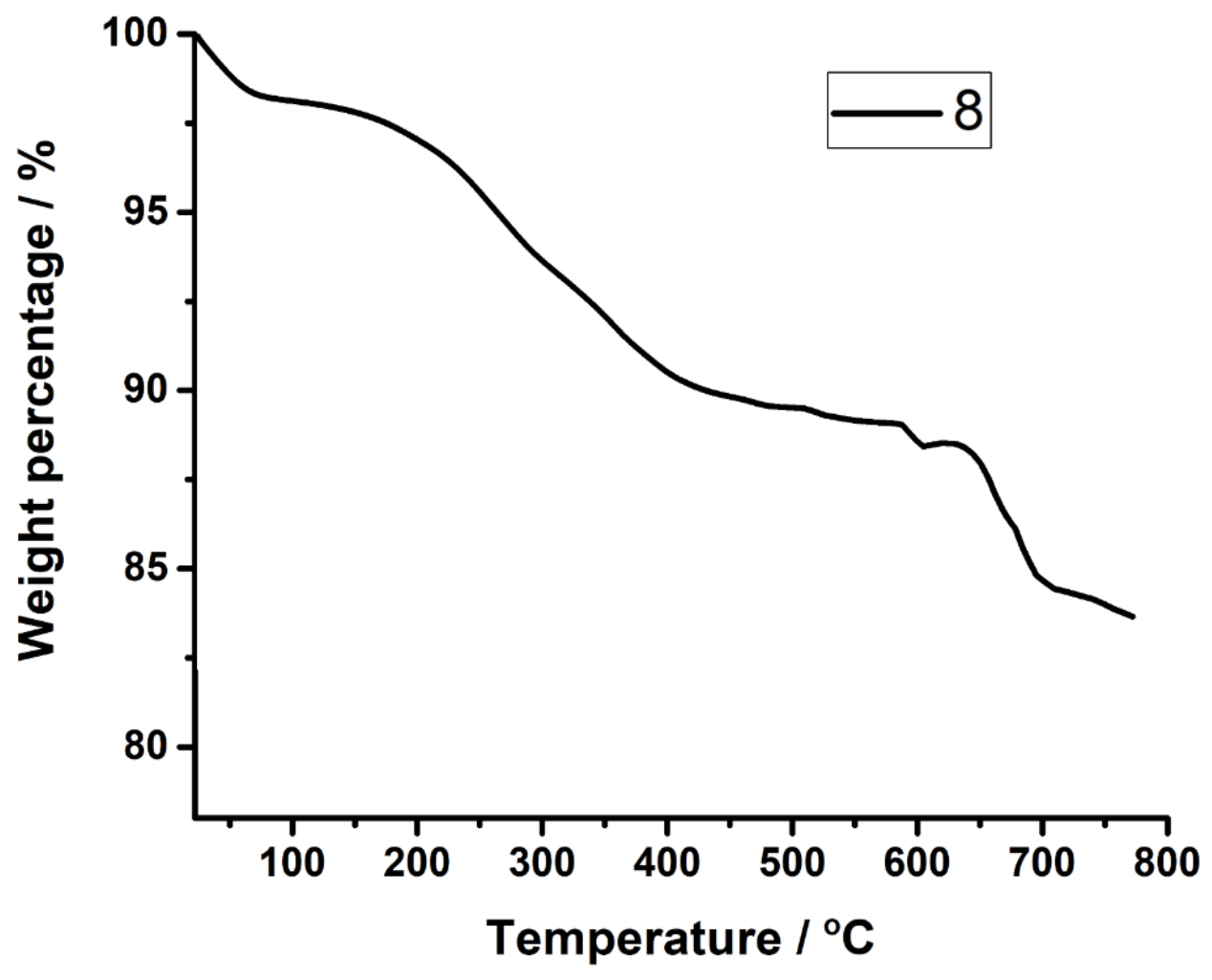

Figure S21. TGA curve for compound 8. 


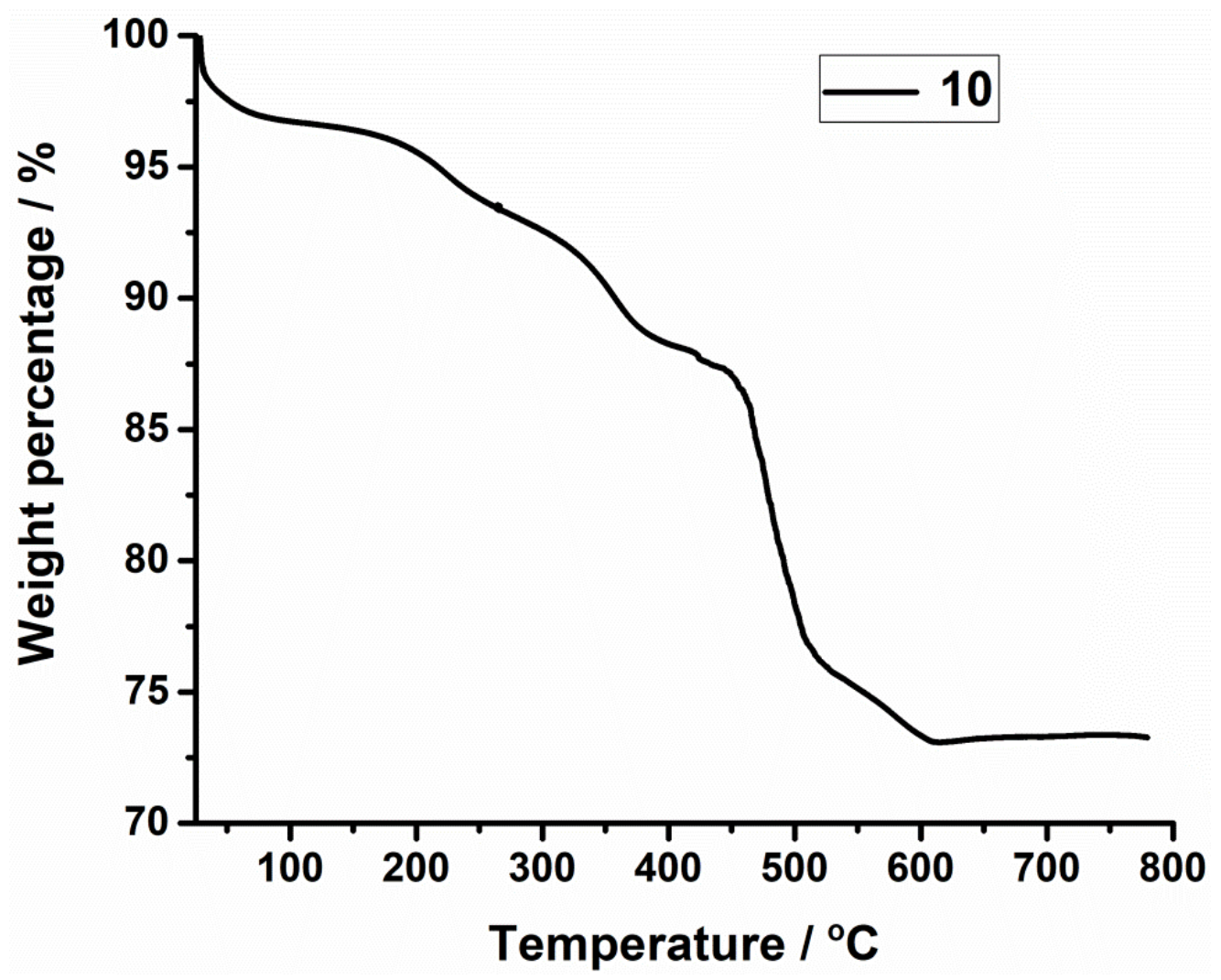

Figure S22. TGA curve for compound 10.

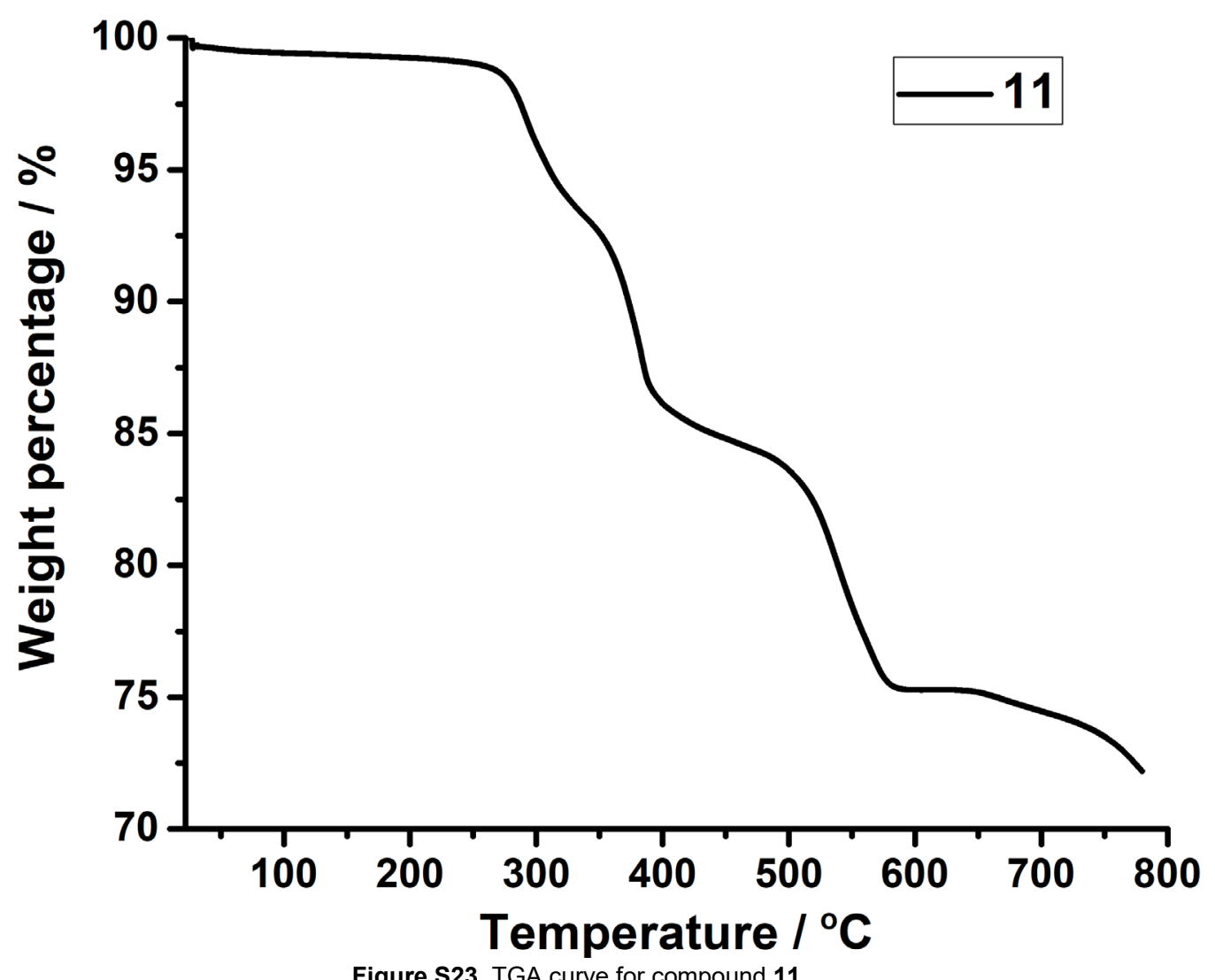

Figure S23. TGA curve for compound 11. 


\section{Redox titrations}

The cerimetric titration was carried out using a $0.0025 \mathrm{M}$ solution of $\mathrm{Ce}^{\mathrm{lV}}$ in $0.5 \mathrm{M}$ of sulphuric acid as oxidant which was added dropwise to a solution of compound 1 (19.2 $\mathrm{mg}$ in $20 \mathrm{~mL}$ of $\mathrm{H}_{2} \mathrm{O}$ ). After addition of $3.1 \mathrm{~mL}$ of the oxidant the colour of the solution turned from deep blue to light yellow along with a characteristic potential jump showed the presence of $1.82 \mathrm{e}^{-}$which (formally) corresponds to $2 \mathrm{Mo}^{\mathrm{V}}$ centers (theoretical value for $2 \mathrm{e}^{-}$reduced species: $3.4 \mathrm{~mL}$ ). The cerimetric titration of all the samples were performed in the same way, and the results are summarized as follows:

\begin{tabular}{ccccc}
\hline Compound & $\begin{array}{c}\text { Concentration } \\
(\mathrm{mg} / 50 \mathrm{~mL})\end{array}$ & $\begin{array}{c}\text { Number of } \\
\text { electrons }\end{array}$ & $\begin{array}{c}\text { Volume of the oxidant } \\
(\mathrm{obs}) \\
(\mathrm{mL})\end{array}$ & $\begin{array}{c}\text { Volume of the oxidant } \\
(\mathrm{cal}) \\
(\mathrm{mL})\end{array}$ \\
\hline $\mathbf{1}$ & 19.2 & 2 & 3.1 & 3.4 \\
$\mathbf{2}$ & 34.8 & 2 & 5.9 & 6.0 \\
$\mathbf{3}$ & 30.0 & 2 & 4.9 & 5.1 \\
$\mathbf{4}$ & 26.0 & 3 & 5.2 & 4.9 \\
$\mathbf{5}$ & 26.0 & 3 & 5.2 & 4.9 \\
$\mathbf{6}$ & 26.0 & 2 & 3.4 & 3.4 \\
$\mathbf{7}$ & 26.0 & 2 & 3.5 & 3.2 \\
$\mathbf{8}$ & 18.0 & 3 & 3.2 & 3.4 \\
$\mathbf{1 0}$ & 16.0 & 4 & 1.5 & 1.4 \\
\hline
\end{tabular}

\section{Reference}

[1] G. Sheldrick, AcataCrystallogr. Sect. A. 1990, 46, 467-473.

[2] G. Sheldrick, ActaCrystallogr. Sect. A, 2008, 64, 112-122.

[3] L. Farrugia, J. Appl. Crystallogr. 1999, 32, 837-838.

[4] R. C. Clark, J. S. Reid, ActaCrystallogr. Sect. A. 1995, 51, 887-897.

[5] A. Dolbecq, J.-D. Compain, P. Mialane, J. Marrot, F. Secheresse, B. Keita, L. Roberto Brudna Holzle, F. Miserque, L. Nadjo, Chem. Eur. J. 2009, 15, 733- 741.

[6] C. Busche, L. Vilà-Nadal, J. Yan, H. N. Miras, D.-L. Long, V. P. Georgiev, A. Asenov, R. H. Pedersen, N. Gadegaard, M. M. Mirza, D. J. Paul, J. M. Poblet, L. Cronin, Nature 2014, 515, 545-549.

[7] Q. Zheng, L. Vilà-Nadal, C. Busche, J. S. Mathieson, D.-L. Long, L. Cronin, Angew. Chemie 2015, 127, 8006-8010; Angew. Chem. Int. Ed. 2015, 54, 7895-7899 